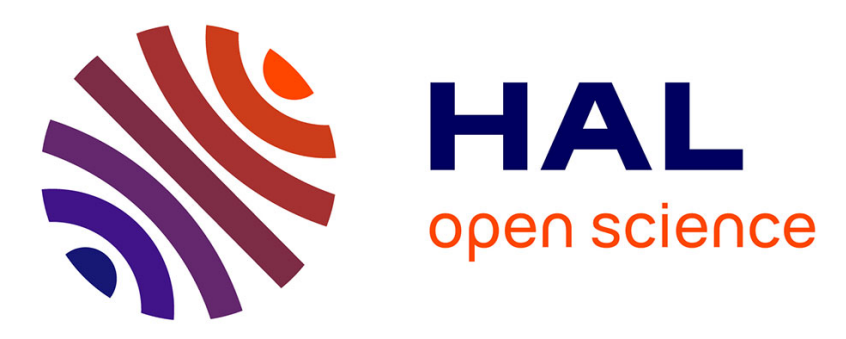

\title{
Selenylated dienes: synthesis, stereochemical studies by 77Se NMR, and transformation into functionalized allenes
}

Sébastien Redon, Anne-Lise Berthe Berkaoui, Xavier Pannecoucke, Francis

Outurquin

\section{To cite this version:}

Sébastien Redon, Anne-Lise Berthe Berkaoui, Xavier Pannecoucke, Francis Outurquin. Selenylated dienes: synthesis, stereochemical studies by 77Se NMR, and transformation into functionalized allenes. Tetrahedron, 2007, 63 (18), pp.3707-3717. 10.1016/j.tet.2007.02.082 . hal-01668240

\author{
HAL Id: hal-01668240 \\ https://hal.science/hal-01668240
}

Submitted on 17 Feb 2018

HAL is a multi-disciplinary open access archive for the deposit and dissemination of scientific research documents, whether they are published or not. The documents may come from teaching and research institutions in France or abroad, or from public or private research centers.
L'archive ouverte pluridisciplinaire HAL, est destinée au dépôt et à la diffusion de documents scientifiques de niveau recherche, publiés ou non, émanant des établissements d'enseignement et de recherche français ou étrangers, des laboratoires publics ou privés. 


\title{
Selenylated dienes: synthesis, stereochemical studies by ${ }^{77}$ Se NMR, and transformation into functionalized allenes
}

\author{
Sébastien Redon, Anne-Lise Berthe Berkaoui, Xavier Pannecoucke* and Francis Outurquin* \\ Université et INSA de Rouen-LHO-IRCOF, UMR 6014 CNRS, 1, rue Tesniere, 76821 Mont Saint-Aignan Cedex, France
}

Received 17 January 2007; revised 19 February 2007; accepted 20 February 2007

Available online 23 February 2007

\begin{abstract}
Phenylselanyl-1,3-dienes 3-8 were prepared by a Wittig or Wittig-Horner-Emmons procedure starting from $\alpha$-phenylselanyl $\alpha, \beta$ unsaturated aldehydes. Ratio and configuration of each diene isomers were determined by ${ }^{77} \mathrm{Se}$ and ${ }^{1} \mathrm{H}$ NMR. These dienes were then oxidized into selenoxides, which could be isolated in some cases. In THF, [2,3]-sigmatropic rearrangement of allylic selenoxides, selenimides, and dihaloselenuranes occurred, yielding allenyl alcohols 12-15, allenyl carbamates 16c-19c, and 1-haloalkyl allenes 20c-22c, respectively.
\end{abstract}

\section{Introduction}

The important place of the 'selenium methodology' in organic synthesis is due to the easy introduction, transformation, and elimination of the selenylated group from organic substrate that allow selective reactions under mild condi-tions. Selenium reagents are now commonly used ${ }^{1-4}$ and extensions to catalysts, ligands, asymmetric synthesis, ${ }^{5}$ and radical chemistry ${ }^{6}$ were the subject of recent works.

In particular, selenoxides play a pivotal role since the facile oxidation of alkyl phenylselenides allows the preparation of olefinic compounds and allylic alcohols under very mild conditions. ${ }^{1,2}$ Our group was mainly involved in the studies of the reactivity of selenylated carbonyl derivatives allowing access to unsaturated, functionalized useful building-blocks ${ }^{7}$ and their transformation into allenic alcohols via oxidation of 2-phenylselanyl-1,3-dienes. ${ }^{8}$ These interesting selenylated dienes could also act as useful intermediates in organic synthesis, for example, as starting materials in Diels-Alder cycloaddition. ${ }^{9,10}$ In the literature, very few preparations of 1-arylselanyl-1,3-butadienes are described: phenylselanyl alkylidene phosphoranes, phenylselanylalk-enals ${ }^{11 \mathrm{a}}$ or palladium-catalyzed cross coupling reactions. ${ }^{1 \mathrm{lb}}$ A stereoselective synthesis of 1,3 -dienyl selenides was re-ported by reaction of $\alpha$-arylselanylvinylstannanes with vinyl halides ${ }^{11 \mathrm{c}}$ or by means of $\alpha$-alkenylzirconium complexes with $\alpha$-phenylselanylvinyl bromides. ${ }^{11 \mathrm{~d}}$

Herein, we present a general access to 2-phenylselanyl-1,3-dienes along with an unambiguous determination of the stereochemistry by ${ }^{77} \mathrm{Se}$ NMR. Due to both vinylic and allylic position of the selanyl group in 2-phenylselanyl-1,3-dienes, sigmatropic rearrangements of selenoxides, selenimides, and dihalo-selenuranes were also studied, yielding, respectively, allenic alcohols, carbamates, and 1-haloalkyl allenes. 


\section{Results and discussion}

Starting from $\alpha, \beta$-unsaturated aldehydes 1 , vinylic $(Z)$ - $\alpha$-phenylselenoaldehydes 2 could be obtained as the unique stereoisomers (except for compound 2c: Z/E 87/13) via a selenenylation process using morpholinobenzeneselen-amide (MBSe) followed by a silica gel 'trap' ${ }^{12}$ Compounds 2 were then transformed into 3-phenylselanyl-1,3-dienes 3-8 via classical Wittig (method A) or Wittig-Horner- Emmons (method B) reactions ${ }^{8}$ (3-6 and 7-8, respectively) (Scheme 1). For clarity reasons, the numbering of selenylated dienes will be 3-phenylselanyl-1,3-dienes in the theoretical part, whereas official numbering will be applied in the experimental part as numbering differs according to substituents.

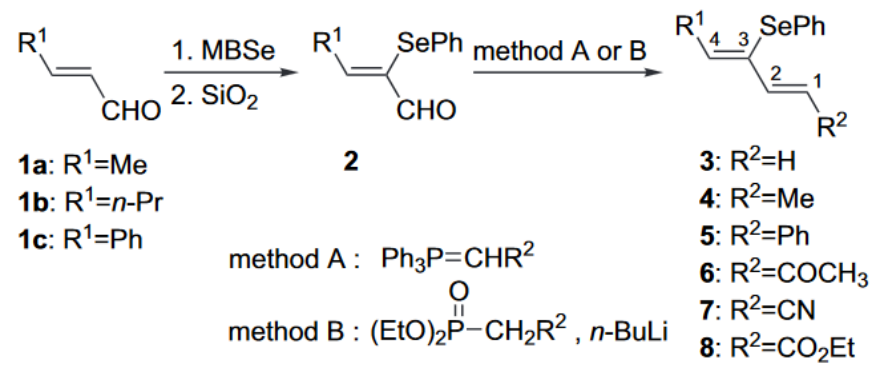

Scheme 1. Synthesis of 3-phenylselanyl-1,3-dienes.

Table 1. Synthesis of conjugated dienes 3-8

\begin{tabular}{|c|c|c|c|c|c|c|c|}
\hline \multirow[t]{2}{*}{ Entry } & \multirow[t]{2}{*}{ No. } & \multirow[t]{2}{*}{$\mathbf{R}^{1}$} & \multirow[t]{2}{*}{$\mathbf{R}^{2}$} & \multirow[t]{2}{*}{ Yields $(\%)\left(\right.$ Method A or B) ${ }^{a}$} & $1 E, 3 Z$ & $\begin{array}{c}1 E, 3 E \\
\text { SeP }\end{array}$ & $1 Z, 3 Z$ \\
\hline & & & & & \multicolumn{3}{|c|}{ Ratio of each diastereomer after chromatography (before chromatography) } \\
\hline 1 & 3a & $\mathrm{Me}$ & $\mathbf{H}$ & 54 (A) & 87 & 13 & \\
\hline 2 & $3 \mathbf{b}$ & Pr & H & 64 (A) & 86 & 14 & \\
\hline 3 & $3 c$ & $\mathrm{Ph}$ & $\mathrm{H}$ & $67(\Lambda)$ & 36 & 64 & \\
\hline 4 & $4 a$ & $\mathrm{Me}$ & $\mathrm{Me}$ & 74 (A) & $35(20)$ & $15(0)$ & $50(80)$ \\
\hline 5 & $4 b$ & $\mathrm{Pr}$ & $\mathrm{Mc}$ & 69 (A) & $48(20)$ & $8(0)$ & $44(80)$ \\
\hline 6 & $4 c$ & $\mathrm{Ph}$ & $\mathrm{Me}$ & 91 (A) & & $40^{\mathrm{b}}(15)$ & $60(70)^{c}$ \\
\hline 7 & $5 \mathbf{a}$ & Me & $\mathrm{Ph}$ & 88 (A) & $70(10)$ & $20(20)$ & $10(70)$ \\
\hline 8 & $5 \mathbf{b}$ & $\operatorname{Pr}$ & $\mathrm{Ph}$ & $82(\mathrm{~A})$ & $70(10)$ & $20(20)$ & $10(70)$ \\
\hline 9 & $5 c$ & $\mathrm{Ph}$ & $\mathrm{Ph}$ & 94 (A) & $55(34)$ & & $45(66)$ \\
\hline 10 & $6 a$ & $\mathrm{Me}$ & COMe & 67 (A) & 80 & 20 & \\
\hline 11 & $6 b$ & $\operatorname{Pr}$ & COMe & $77(\Lambda)$ & 80 & 20 & \\
\hline 12 & $6 c$ & $\mathrm{Ph}$ & COMe & 88 (A) & 55 & 45 & \\
\hline 13 & $7 a$ & $\mathrm{Me}$ & $\mathrm{CN}$ & 61 (B) & $68(68)$ & $10(10)$ & $20^{d}(23)$ \\
\hline 14 & $7 \mathbf{b}$ & $\mathrm{Pr}$ & $\mathrm{CN}$ & 82 (B) & $68(68)$ & $10(10)$ & $20^{\mathrm{d}}(23)$ \\
\hline 15 & $7 c$ & $\mathrm{Ph}$ & $\mathrm{CN}$ & 83 (B) & $48^{\mathrm{b}}(48)$ & 20 (14) & $30^{d}(38)$ \\
\hline 16 & $8 \mathrm{a}$ & $\mathrm{Mc}$ & $\mathrm{CO}_{2} \mathrm{Et}$ & 72 (B) & 80 & 20 & \\
\hline 17 & $8 b$ & $\mathrm{Pr}$ & $\mathrm{CO}_{2} \mathrm{Et}$ & 71 (B) & 80 & 20 & 0 \\
\hline 18 & $8 c$ & $\mathrm{Ph}$ & $\mathrm{CO}_{2} \mathrm{Et}$ & 84 (B) & $84^{\mathrm{b}}(54)$ & $16(46)$ & \\
\hline
\end{tabular}

"Sec Scheme 1.

b X-ray analysis could be obtained for this isomer.

c $15 \%$ of $17,3 E$ isomer.

d Traces of $1 Z, 3 E$ isomer.

Only dienes $8\left(\mathrm{R}^{2}=\mathrm{CO}_{2} \mathrm{Et}\right)$ and $\mathbf{5 c}\left(\mathrm{R}^{1}=\mathrm{R}^{2}=\mathrm{Ph}\right)$ were al-ready synthesized by our group. ${ }^{8}$ In this paper, we wanted to extend this methodology to the preparation of various functionalized dienes 6-7 and to less stabilized one 3-5. The yields were ranging from $45 \%$ to $94 \%$ for method $\mathrm{A}$ and from $61 \%$ to $84 \%$ for method B (Table 1). Indeed, the creation of the C1-C2 double bond (see Scheme 1) gave of-ten a mixture of $Z / E$ diastereomers, some of them were not stable and partial isomerization occurred during column chromatography on silica gel.

Wittig reactions with non-stabilized ylides $\left(\mathrm{R}^{2}=\mathrm{Me}\right)$ formed predominantly $Z$ isomer of the $\mathrm{C} 1-\mathrm{C} 2$ double bond (Table 1 , entries 4-6). C1-C2 configurations were easily determined by measurement of ${ }^{3} J_{\mathrm{H} 1-\mathrm{H} 2}$ coupling constant $(11.0-12.0 \mathrm{~Hz}$ for $Z$ and $14.6-15.6 \mathrm{~Hz}$ for $E)$. With semi-stabilized ylides $\left(\mathrm{R}^{2}=\mathrm{Ph}\right.$, Table 1, entries 7-9), NMR spectrum of the crude reaction shows three diastereomers, the major one having $\mathrm{Z}$ configuration at $\mathrm{C} 1-\mathrm{C} 2$ double bond $(70 \%$ for $\mathbf{5 a}$ and $\mathbf{5 b}, 66 \%$ for $\mathbf{5 c})$. In both cases, $Z$ configuration for $\mathrm{C} 1-\mathrm{C} 2$ double bond is not stable and isomerization occurred during silica gel 
chromatography, modifying the diastereomer ratio and rendering their separation difficult. Heating of diastereomeric mixtures led to a complete transformation of the $\mathrm{C} 1-\mathrm{C} 2 Z$ isomer into the thermodynamically more stable $E$ isomer (entry 4 : $1 E, 3 Z / 1 E, 3 E: 70 / 30$; entry 5: $1 E, 3 Z / 1 E, 3 E: 76 / 24)$. With stabilized ylides, Wittig or Wittig-Horner-Emmons reactions afforded preferentially $\mathrm{C} 1-\mathrm{C} 2 E$ isomer $\left(6 \mathrm{R}^{2}=\mathrm{COMe}: 100 \%, 7 \mathrm{R}^{2}=\mathrm{CN}: 68-77 \%, \mathbf{8} \mathrm{R}^{2}=\mathrm{CO}_{2} \mathrm{Et}: 100 \%\right)$. For all the ylides, we observed partial C3-C4 isomerization as starting from $Z$ selenylated enal 2, we always ended up with C3-C4 $E$ and $Z$ isomers (NMR analysis of crude material).

Due to the complex mixture of diastereomers obtained in the olefination reaction $(\mathrm{C} 1-\mathrm{C} 2$ and $\mathrm{C} 3-\mathrm{C} 4$ isomerizations during the purification steps and the reaction time, respectively), determination of the $\mathrm{C} 3-\mathrm{C} 4$ double bond configuration became quickly a necessity (Table 1). Due to the absence of hydrogen at $\mathrm{C} 3$ position and a $\mathrm{H} 4$ signal often overlapped on Ar-H signals in ${ }^{1} \mathrm{H}$ NMR spectra, determination of the configurations was very difficult even with NOESY experiment. ${ }^{77}$ Se NMR appeared to be the easiest and the more reliable method to answer this question. In-deed with a single spectrum, we could determine the ratio and the configuration of each selenylated diene isomers (Table 2). When $\mathrm{C} 1-\mathrm{C} 2$ double bond is $E$, ${ }^{77} \mathrm{Se}$ NMR chemical shift is typical of diene configuration $(1 \mathrm{E}, 3 \mathrm{E}: 375.8<\delta<423.2 ; 1 \mathrm{E}, 3 \mathrm{Z}: 251.8<\delta<308.5)$. In the same series, chemical shift of $\mathrm{H} 2$ in ${ }^{1} \mathrm{H}$ NMR is always $0.3 \mathrm{ppm}$ lower for $3 Z$ compared to $3 E$ isomer. Configurations $1 E, 3 Z$ of dienes $7 \mathbf{c}$ and $\mathbf{8 c}$ and $1 E, 3 E$ of $\mathbf{4 c}$ were confirmed by X-ray diffraction.

When $\mathrm{C} 1-\mathrm{C} 2$ double bond configuration is $\mathbf{Z}$, the ${ }^{77} \mathrm{Se}$ NMR chemical shift (1Z,3Z: $348.5<\delta<415.4$ ) is difficult to com-pare with $\mathrm{C} 1-\mathrm{C} 2 E$ series. Indeed in the $Z$ series, diene seems to adopt $\mathrm{S}$-cis conformation as opposed to the $\mathrm{C} 1-\mathrm{C} 2 E$ series $(S$ trans). This would be in total accordance with a study of 'Conformational thermodynamic and kinetic parameters of methylsubstituted 1,3-butadienes', which showed the steric interactions in the 1,3-diene framework. ${ }^{14}$ Indeed in the $\mathrm{C} 1-\mathrm{C} 2 \mathrm{Z}$ series, strong interaction between $\mathrm{SePh}$ and $\mathrm{R}^{2}$ under S-trans conformation forced the dienes to adopt a $\mathrm{S}$-cis conformation. Unfortunately, due to mixture and whatever the NOESY experimental parameters were, we were unable to prove or disprove this assumption.

Table 2. ${ }^{77}$ Se and 'H NMR data (ppm) of dienes 3-8

\begin{tabular}{|c|c|c|c|c|c|c|c|c|c|}
\hline \multirow[t]{2}{*}{ Entry } & \multirow[t]{2}{*}{ No. } & \multirow[t]{2}{*}{$\mathrm{R}^{3}$} & \multirow[t]{2}{*}{$\mathrm{R}^{2}$} & \multicolumn{2}{|c|}{$1 E, 3 Z$} & \multicolumn{4}{|c|}{$1 Z, 3 Z$} \\
\hline & & & & ${ }^{77} \mathrm{Se}$ & ${ }^{1} \mathbf{H}(\mathbf{H}-2)$ & ${ }^{77} \mathrm{Se}$ & ${ }^{1} \mathrm{H}(\mathrm{H}-2)$ & ${ }^{77} \mathrm{Sc}$ & ${ }^{1} \mathrm{H}(\mathrm{H}-2)$ \\
\hline 1 & $3 a^{a}$ & $\mathrm{Mc}$ & $\mathrm{H}$ & 251.8 & 6.40 & 387.4 & 6.69 & & \\
\hline 2 & $3 \mathbf{b}$ & $\operatorname{Pr}$ & H & 260.3 & 6.39 & 375.8 & 6.67 & & \\
\hline 3 & $3 e^{a}$ & $\mathrm{Ph}$ & $\mathrm{H}$ & 297.1 & 6.51 & 409.2 & 6.83 & & \\
\hline 4 & $4 \mathbf{a}$ & $\mathrm{Mc}$ & $\mathrm{Mc}$ & 265.1 & 6.12 & 399.8 & 6.39 & 362.7 & 5.80 \\
\hline 5 & $4 b$ & $\operatorname{Pr}$ & $\mathrm{Me}$ & 273.3 & 6.10 & 400.8 & 6.37 & 369.2 & 5.82 \\
\hline 6 & $4 c$ & $\mathrm{Ph}$ & $\mathrm{Me}$ & & & 418.5 & 6.56 & 411.0 & 5.86 \\
\hline 7 & $5 \mathbf{a}$ & $\mathrm{Mc}$ & $\mathrm{Ph}$ & 261.7 & 7.01 & 396.3 & 7.13 & 368.6 & 6.14 \\
\hline 8 & $5 b$ & $\operatorname{Pr}$ & $\mathrm{Ph}$ & 269.8 & 6.97 & 397.9 & nd & 374.9 & 6.16 \\
\hline 9 & $5 c$ & $\mathrm{Ph}$ & $\mathrm{Ph}$ & 304.0 & nd & & & 415.4 & 6.18 \\
\hline 10 & $6 a$ & $\mathrm{Mc}$ & COMe & 267.4 & nd & 401.6 & 7.56 & & \\
\hline 11 & $6 b$ & Pr & COMe & 275.6 & 7.22 & 403.7 & 7.54 & & \\
\hline 12 & $6 c$ & $\mathrm{Ph}$ & COMe & 308.5 & nd & 423.2 & 7.61 & & \\
\hline 13 & $7 \mathbf{a}$ & $\mathrm{Me}$ & $\mathrm{CN}$ & 252.7 & 7.09 & 385.8 & 7.40 & 348.5 & 6.67 \\
\hline 14 & $7 b$ & $\operatorname{Pr}$ & $\mathrm{CN}$ & 260.7 & 7.10 & 387.1 & nd & 357.3 & 6,69 \\
\hline 15 & $7 e$ & $\mathrm{Ph}$ & $\mathrm{CN}$ & 288.2 & nd & 408.6 & nd & 398.4 & 6.75 \\
\hline 16 & $8 a^{a}$ & $\mathrm{Me}$ & $\mathrm{CO}_{2} \mathrm{Et}$ & 263.6 & 7.41 & 399.6 & 7.72 & & \\
\hline 17 & $8 b$ & $\operatorname{Pr}$ & $\mathrm{CO}_{2} \mathrm{Et}$ & 271.5 & 7.41 & 401.1 & 7.70 & & \\
\hline 18 & $8 e$ & $\mathrm{Ph}$ & $\mathrm{CO}_{2} \mathrm{Et}$ & 301.1 & 7.55 & 421.6 & 7.80 & & \\
\hline
\end{tabular}

The only sulfur analogues found in the literature confirm configurations and d ${ }^{1} \mathrm{H},{ }^{13} \mathrm{C}$ NMR of dienes $\mathbf{3 a},{ }^{13 \mathrm{a}} \mathbf{3 c},{ }^{13 \mathrm{~b}}$ and $\mathbf{8 a} .{ }^{13 \mathrm{c}}$

The olefination reaction could be resumed as follows (Scheme 2: with non- and semi-stabilized ylides; Scheme 3: with stabilized ylides).

With non- or semi-stabilized ylides, olefination reaction on selenylated enal $\mathbf{2}$ was stereochemically controlled to yield mainly $1 Z$ isomer (Scheme 2). Under the reaction condi-tions, partial C3-C4 isomerization occurred, delivering $3 E$ isomer. Due to structural steric instability (S-cis conforma-tion), $\mathrm{C} 1-\mathrm{C} 2 Z$ isomer isomerized to afford more stable $1 E$ isomers (Table 1: entries 4-9). 
With stabilized ylides, as it could be predicted, the stereo-chemistry of the created double bond C1-C2 was $E$ : exclu-sively when $\mathrm{R}^{2}=\mathrm{COMe}$ or $\mathrm{CO}_{2} \mathrm{Et}$ (Table 1: entries 10-12, 16-18), as the major isomer with $\mathrm{R}^{2}=\mathrm{CN}$ (Table 1: entries 13-15). Under the reaction conditions, as for non- or semi-stabilized ylides, partial $\mathrm{C} 3-\mathrm{C} 4$ isomerization occurred, delivering $3 E$ isomer (Scheme 2).

Scheme 2. Olefination reaction with non- and semi-stabilized ylides.

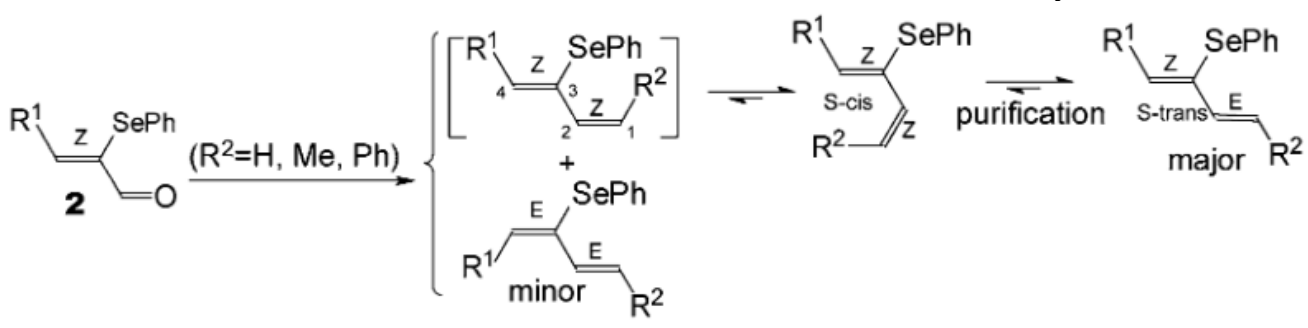

\subsection{Allenes formation}

Allenic compounds have been the subject of intensive studies. ${ }^{15}$ Due to the versatile functionality of 1,2-diene moiety, allenes could serve as either a nucleophile or an electrophile, allowing them to act as precursors for cyclo-addition reactions. ${ }^{16}$ In particular, palladium-catalyzed reactions of allenes have gained considerable attention in recent years. ${ }^{17,18}$ Concerning their formation, allenes are of-ten prepared via addition of organometallic reagents (essentially cuprates) to enynes or to propargylic electrophiles via an $\mathrm{S}_{\mathrm{N}} 2^{0}$ reaction. ${ }^{19}$ Several methods exist to prepare $\alpha$-hydroxyallenes: addition of lithiated allenes to aldehydes or ketones; ${ }^{20,21}$ hydride reduction or Grignard addition onto propargylic oxiranes; ${ }^{16,22}[2,3]$-Wittig rearrangement of propargyl ethers; ${ }^{23}$ insertion of alkenylidene carbenes into the $\alpha \mathrm{C}-\mathrm{H}$ bond of alkoxides. ${ }^{24}$

We present a new and mild method that is compatible with various chemical functionalities to prepare $\alpha$-hydroxy-allenes from selenylated 1,3-dienes after oxidation with hydrogen peroxide. First experiments were performed with stabilized 1,3-dienes 3c-8c derived from cinnamaldehyde. The selenium atom at allylic position was first oxidized, affording selenoxides, which further rearrange into $\alpha$-hydroxyallenes $\mathbf{1 2 c - 1 5 c}$ (Table 3). When $R^{2}$ is an electron

Table 3. $\alpha$-Hydroxyallenes' synthesis

\begin{tabular}{|c|c|c|c|}
\hline$=L^{\mathrm{SePh}}$ & $\frac{\mathrm{H}_{2} \mathrm{O}_{2}}{\mathrm{CH}_{2} \mathrm{Cl}_{2}, \mathrm{r}}$ & $\underbrace{S e=0}_{9 c-11 c} \frac{\mathrm{THF}}{\mathrm{R}^{2}}$ & $\stackrel{\mathrm{Ph}}{=} \underbrace{}_{1 \mathrm{HO}}-\mathrm{R}^{2}$ \\
\hline Starting dienes & $\mathrm{R}^{2}$ & Selenoxides (yield \%) & Allenes (yield \%) \\
\hline $3 c$ & H & - & $12 c(78)$ \\
\hline $4 c$ & $\mathrm{Me}$ & - & $13 \mathrm{c}(81)$ \\
\hline $5 c$ & $\mathrm{Ph}$ & - & $14 \mathbf{c}(81)$ \\
\hline 6c & $\mathrm{COCH}_{3}$ & $9 c(81)$ & - \\
\hline $7 \mathrm{c}$ & & $10 c(63)$ & \\
\hline $8 c$ & $\mathrm{CO}_{2} \mathrm{Et}$ & $11 \mathrm{c}(76)$ & $15 c(75)$ \\
\hline
\end{tabular}

In all cases, the rearrangement into $\alpha$-hydroxyallenes was conducted in THF in the presence of triethylamine at room temperature to give compounds 12c-15c in good yields (Table 3). For compound $12 \mathrm{c},{ }^{25}$ only one diastereomer was detected by ${ }^{13} \mathrm{C}$ NMR, but two isomers are present in the case of allenes 13c $(63 / 37), \mathbf{1 4}^{8}(70 / 30)$, and $\mathbf{1 5} \mathbf{c}^{8}(70 / 30)$. Noteworthy the [1,3]-sigmatropic rearrangement of conjugated dienes is specific to selenoxides, no rearrangement occurred with sulfoxides. However, when $\mathrm{R}^{2}$ was a carbonyl or a nitrile group, the selenoxides $9 \mathbf{c}$ and $\mathbf{1 0 c}$ decomposed, whatever the conditions, and we were unable to isolate allenes.

After variation of $\mathrm{R}^{2}$ group, we studied the influence of $\mathrm{R}^{1}$ group on the transformation of 1,3-dienes into $\alpha$ - hydroxyallenes via [1,3]-sigmatropic rearrangement of allylic selenoxides. For this, our group ${ }^{8}$ has already shown that when $\mathrm{R}^{2}$ is COOEt, the yield was much better when $R^{1}$ is an aryl than an alkyl group (Table 4: compounds 15a-c). 
Table 4. $\alpha$-Hydroxyallenes' synthesis: influence of $R^{1}$ group

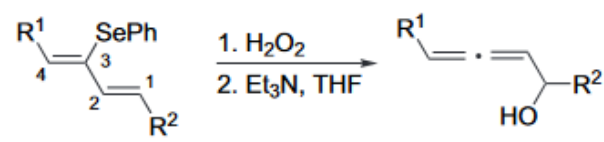

\begin{tabular}{lllc}
\hline Starting dienes & $\mathrm{R}^{1}$ & $\mathrm{R}^{2}$ & Allenes (yield \%) \\
\hline $\mathbf{4 a}$ & $\mathrm{Me}$ & $\mathrm{Me}$ & $\mathbf{1 3 a}^{26}(51)$ \\
$\mathbf{4 b}$ & $n-\mathrm{Pr}$ & $\mathrm{Me}$ & $\mathbf{1 3 b}(63)$ \\
$\mathbf{4 c}$ & $\mathrm{Ph}$ & $\mathrm{Me}$ & $\mathbf{1 3 c}(81)$ \\
$\mathbf{5 a}$ & $\mathrm{Me}$ & $\mathrm{Ph}$ & $\mathbf{1 4 a}^{27}(38)$ \\
$\mathbf{5 b}$ & $n-\mathrm{Pr}$ & $\mathrm{Ph}$ & $\mathbf{1 4 b}(67)$ \\
$\mathbf{5 c}$ & $\mathrm{Ph}$ & $\mathrm{Ph}$ & $\mathbf{1 4 c}^{8}(81)$ \\
$\mathbf{8 a}$ & $\mathrm{Me}$ & $\mathrm{CO}_{2} \mathrm{Et}$ & $\mathbf{1 5 a}^{8}(30)$ \\
$\mathbf{8 b}$ & $n-\mathrm{Pr}$ & $\mathrm{CO}_{2} \mathrm{Et}$ & $\mathbf{1 5 b}^{8}(30)$ \\
$\mathbf{8 c}$ & $\mathrm{Ph}$ & $\mathrm{CO}_{2} \mathrm{Et}$ & $\mathbf{1 5 c}^{8}(75)$ \\
\hline
\end{tabular}

Complementary results showed that when $\mathrm{R}^{1}$ is an alkyl or phenyl group regardless of the nature of $\mathrm{R}^{2}$ : methyl or phenyl, the $\alpha$ hydroxyallenes 13 and 14 could be obtained with good yields (see Table 4). Furthermore, yields were always higher when $\mathrm{R}^{1}$ changed by following the order phenyl>propyl>methyl. In all the cases cited above, two diastereomers of allene were detected by ${ }^{13} \mathrm{C}$ NMR. Further studies are still undertaken to try to understand and to explain the stereoselectivity.

withdrawing group, the moderately stable selenoxides could be isolated as a mixture of diastereomers (Table 3: compounds 9c, 10c, and 11c). The stereochemistry of these selenoxides was impossible to attribute due to complex NMR spectra (as starting dienes were also mixture of isomers, vide supra) and instability of the products.

Due to their pharmaceutical interests (anesthetic, hypnotic, enzyme inhibitors ${ }^{28}$ or treatment of depression ${ }^{29}$ ), numerous methods for the preparation of allenic carbamates are de-scribed in the literature,${ }^{15-18}$ but none of them have exploited the mildness and the selectivity of organoselenium chemistry.

Hopkins and co-workers ${ }^{30}$ studied the [2,3]-sigmatropic re-arrangement of allylic selenimides and nitrogen analogues of selenoxides. Thus, $\mathrm{N}$-allyl carbamates were prepared by oxidation with anhydrous $N$-chlorosuccinimide (NCS) in methanol in the presence of tert-butyl carbamate.

We applied this reaction to dienes $3 \mathbf{c}, \mathbf{4 c}, \mathbf{5 c}$, and $\mathbf{8 c}$ (Scheme 4). After oxidation by $N$-chlorosuccinimide in the presence of tertbutyl carbamate, rearrangement of the selenimide followed by hydrolysis of the intermediate selenamide, we ended up, respectively, with allenic carbamates $16 \mathbf{c}, \mathbf{1 7 c}, \mathbf{1 8 c}$, and 19c (in 43-53\% yields). It is significant to note that traces of water can induce partial hydrolysis of the selen-imide, being able to lead to the formation of corresponding hydroxyallene. ${ }^{1} \mathrm{H}$ and ${ }^{13} \mathrm{C}$ NMR studies showed the pres-ence of only one diastereomer presumably due to an overlap of the chemical shifts of the two expected diastereomers.

Finally, to complete the studies concerning the transformation of easily accessible 3-selenylated-1,3-dienes into various $\alpha$ functionalized allenes species, chlorination and bromination of selenylated conjugated dienes were undertaken (Scheme 4). Dihalogenated adducts, obtained from halogenation of conjugated dienes, were decomposed in $\mathrm{CCl}_{4}$ under reflux, yielding $\alpha$ halogenated allenes. The allenes could be obtained only, if ethylvinylether, a $\mathrm{PhSeX}$ trap, is present in the reaction mixture. This additive is necessary to shift the equilib-rium to the formation of allenes.

Scheme 4. Allenyl carbamates and 1-halogenoalkyl allenes syntheses. ${ }^{31}$

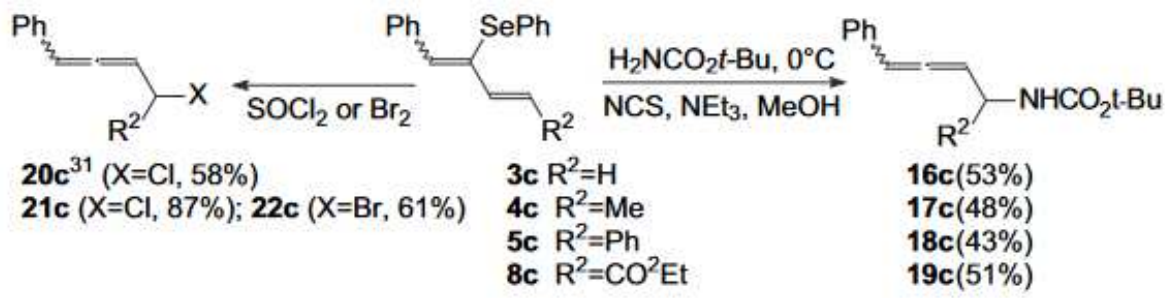

In conclusion, we have developed a general access to 2-phenylselanyl-1,3-dienes and an unambiguous determination of the stereochemistry of the two double bonds based on ${ }^{77} \mathrm{Se}$ NMR. These dienes, due to both vinylic and allylic position of selanyl group could be transformed via [1,3]-sigmatropic rearrangements into variously functionalized allenes.

This transformation occurred via selenoxide, selenimide or halo-selenurane intermediates under mild and selective conditions that give access to even none stabilized a-hydroxy, $\alpha$-amino or $\alpha$-halogenoallenes. 


\section{Experimental}

\subsection{General}

Solvents were purified by conventional methods prior to use. TLC was performed on Merck 60F-250 silica gel plates and column chromatography over silica gel SI 60 (230-240 mesh). Melting points were taken on a Kofler apparatus and were uncorrected. Elemental analyses were carried out on a Carlo Erba EA 1100 analyzer and mass spectra on a HP5890 (electronic impact $70 \mathrm{eV}$ ) using GC-MS coupled with a Jeol AX 500. NMR spectra were recorded on a Bruker DPX 300 spectrometer operating at $300 \mathrm{MHz}$ for proton and $75.4 \mathrm{MHz}$ for carbon. This probe is equipped with pulsed-field (z) gradients. ${ }^{77} \mathrm{Se}$ NMR spectra were recorded at $21 \mathrm{C}$ on a Bruker DPX 400 spectrometer operating at $76.29 \mathrm{MHz}$ for ${ }^{77} \mathrm{Se}$, using a pulse length of $19 \mu \mathrm{s}$ ( 90 pulse $=19 \mu \mathrm{s}$ ) and an optimized relaxation delay of $2 \mathrm{~s}$. An average of $1500 \mathrm{scans}$ for ${ }^{77} \mathrm{Se}$ NMR was necessary to have reliable information. Chemical shifts (d) are expressed in ppm relative to TMS for ${ }^{1} \mathrm{H}$ and ${ }^{13} \mathrm{C}$ nuclei and to $\mathrm{Me}_{2} \mathrm{Se}$ for ${ }^{77} \mathrm{Se}$ nuclei; coupling constants ( $\mathrm{J}$ ) are given in Hertz; coupling multiplicities are reported using conventional abbreviations.

3.1.1. Preparation of $\alpha$-phenylselanyl enals $\mathbf{2}$. A solution of enal $\mathbf{1}(10 \mathrm{mmol})$ in anhydrous dichloromethane $(50 \mathrm{ml})$ was added dropwise to the solution of $N$-phenylselanylmor-pholine ${ }^{12 a}(1.8 \mathrm{~g}, 20 \mathrm{mmol})$, in the same solvent $(20 \mathrm{ml})$.

The mixture was stirred for $6 \mathrm{~h}$ at room temperature and then hydrolyzed by $1 \mathrm{~N} \mathrm{HCl}$ solution $(15 \mathrm{ml})$. The aqueous layer was extracted with dichloromethane $(3 \times 50 \mathrm{ml})$ and the organic fractions were dried and concentrated. The $\alpha$-phenylselanyl enals 2 were purified by chromatography on silica gel (elution with a mixture of petroleum ether/ $\mathrm{CH}_{2} \mathrm{Cl}_{2}: 85 / 15$ ).

3.1.1.1. (Z)-2-Phenylselanylbut-2-enal 2a. Yield $85 \%$, oil, ${ }^{77} \mathrm{Se} \delta$ (ppm) $261.0 ;{ }^{1} \mathrm{H}$ NMR $\delta$ (ppm) 2.18 (d, $3 \mathrm{H}, J=6.7 \mathrm{~Hz}$ ), 7.20-7.27 (m, 3H), 7.32-7.40 (m, 3H), 9.41 (s, 1H, H-1); ${ }^{13} \mathrm{C}$ NMR $\delta$ (ppm) 19.1, 127.1, 129.3, 129.5, 129.6, 131.8, 135.3, 137.3 (C-2), 158.0 (C-3), 190.9 (C-1).

3.1.1.2. (Z)-2-Phenylselanylhex-2-enal 2b. Yield 83\%, oil, ${ }^{77} \mathrm{Se} \delta(\mathrm{ppm}) 268.5$; ${ }^{1} \mathrm{H}$ NMR $\delta$ (ppm) 0.97 (t, $3 \mathrm{H}, J=7.4 \mathrm{~Hz}$ ), $1.55(\mathrm{~m}, 2 \mathrm{H}), 2.60$ (q, 2H, J=7.2 Hz), 7.20-7.29 (m, 3H), 7.26 (t, 1H, J=7.2 Hz, H-3), 7.37-7.41 (m, 2H), 9.40 (s, 1H, H-1); ${ }^{13} \mathrm{C}$ NMR $\delta$ (ppm) 14.0, 21.7, 34.8, 127.1, 129.3, 129.8, 131.9, 136.1 (C-2), 162.5 (C-3), 191.0 (C-1).

3.1.1.3. 3-Phenyl-2-phenylselanylprop-2-enal 2c. Yield 88\%, Z/E 87/13. (Z)-isomer: $\mathrm{mp}=64{ }^{\circ} \mathrm{C},{ }^{77} \mathrm{Se} \delta$ (ppm) $301.1 ;{ }^{1} \mathrm{H}$ NMR $\delta(\mathrm{ppm}) 7.18-7.26(\mathrm{~m}, 3 \mathrm{H}), 7.38-7.44(\mathrm{~m}, 5 \mathrm{H}), 7.84-7.88(\mathrm{~m}, 2 \mathrm{H}), 8.01(\mathrm{~s}, 1 \mathrm{H}, \mathrm{H}-3), 9.50(\mathrm{~s}, 1 \mathrm{H}, \mathrm{H}-1)$;

${ }^{13} \mathrm{C}$ NMR $\delta$ (ppm) 127.4, 128.6, 129.4, 131.0, 131.2, 132.1, 132.5 (C-2), $152.6(\mathrm{C}-3), 191.6(\mathrm{C}-1)$. (E)-isomer: oil, ${ }^{77} \mathrm{Se} \mathrm{d}$ (ppm) 409.5; ${ }^{1} \mathrm{H}$ NMR $\delta(\mathrm{ppm}) 7.18-7.24(\mathrm{~m}, 3 \mathrm{H}), 7.32$ (s, 1H, H-3), 7.34-7.42 (m, 5H), 7.64-7.69 (m,2H), 9.79 (s, 1H, H1 ); ${ }^{13}$ C NMR $\delta$ (ppm) 126.6, 128.7, 129.1, 129.4, 129.6, 129.9, 134.8, 136.5, 138.5 (C-2), 145.5 (C-3), 189.1 (C-1).

3.1.2. Preparation of 3-phenylselanyl-1,3-dienes 3. At room temperature, a solution of $n$-BuLi in hexanes (1.6 M, $3.45 \mathrm{ml}$, $5.5 \mathrm{mmol}$ ) was slowly added, under argon, to methyltriphenylphosphonium bromide (1.96 g, $5.5 \mathrm{mmol})$ in anhydrous THF (30 ml). After stirring for $0.5 \mathrm{~h}, \alpha$-phenyl-selanyl enal $2(5 \mathrm{mmol})$ in THF $(5 \mathrm{ml})$ was added dropwise. The mixture was stirred for $3 \mathrm{~h}$ under reflux, quenched with saturated aqueous $\mathrm{NH}_{4} \mathrm{Cl}$, and extracted with diethylether. The organic extracts were dried over magnesium sulfate and concentrated under vacuum. The residue was purified by chromatography on silica gel (light petroleum) and rectified by Kugelrhor distillation.

3.1.2.1. 3-Phenylselanylpent-1,3-diene 3a. Yield 54\%, Z/E 87/13. (Z)-isomer: oil, ${ }^{77} \mathrm{Se} \delta$ (ppm) 251.8; ${ }^{1} \mathrm{H}$ NMR $\delta$ (ppm) $2.00(\mathrm{~d}, 3 \mathrm{H}, J=6.7 \mathrm{~Hz}, \mathrm{H}-5), 5.06\left(\mathrm{~d}, \mathrm{H}_{\mathrm{cis}}, J=10.2 \mathrm{~Hz}\right), 5.59$ (d, $\left.\mathrm{H}_{\text {trans }}, J=16.5 \mathrm{~Hz}\right), 6.32(\mathrm{q}, 1 \mathrm{H}, J=6.7 \mathrm{~Hz}, \mathrm{H}-4), 6.40(\mathrm{dd}, 1 \mathrm{H}$, $J=10.2,16.5 \mathrm{~Hz}, \mathrm{H}-2), 7.10-7.32(\mathrm{~m}, 5 \mathrm{H}) ;{ }^{13} \mathrm{C}$ NMR d (ppm) $18.4(\mathrm{Me}), 117.1$ (C-1), 125.9, 129.1, 129.3, 129.9, 131.6 (C3), 138.5 (C-2), 138.8 (C-4). Anal. Calcd for $\mathrm{C}_{11} \mathrm{H}_{12} \mathrm{Se}: \mathrm{C}, 59.20$; H, 5.42. Found: C, 58.96; H, 5.37. (E)-isomer: oil, ${ }^{77} \mathrm{Se} \delta$ (ppm) 387.4; ${ }^{1} \mathrm{H}$ NMR $\delta(\mathrm{ppm}) 1.91(\mathrm{~d}, 3 \mathrm{H}, J=7.2 \mathrm{~Hz}, \mathrm{H}-5), 5.23$ (d, $\left.\mathrm{H}_{\text {cis }}, J=10.8 \mathrm{~Hz}\right), 5.64\left(\mathrm{~d}, \mathrm{H}_{\text {trans }}, J=16.7 \mathrm{~Hz}\right), 6.02(\mathrm{q}$, $1 \mathrm{H}, J=6.7 \mathrm{~Hz}, \mathrm{H}-4), 6.69$ (dd, 1H, J=10.8, $16.7 \mathrm{~Hz}, \mathrm{H}-2), 7.10-7.32(\mathrm{~m}, 5 \mathrm{H}) ;{ }^{13} \mathrm{C}$ NMR $\delta(\mathrm{ppm}) 18.0(\mathrm{Me}), 119.9(\mathrm{C}-1)$, $127.8,129.1,129.3,131.2,131.6(\mathrm{C}-3), 137.1(\mathrm{C}-2), 137.9(\mathrm{C}-4)$.

3.1.2.2. 3-Phenylselanylhept-1,3-diene 3b. Yield 64\%, Z/E 86/14. (Z)- isomer: oil, ${ }^{77} \mathrm{Se} \delta$ (ppm) $260.3 ;{ }^{1} \mathrm{H}$ NMR d (ppm) $0.91(\mathrm{t}, 3 \mathrm{H}, J=7.4 \mathrm{~Hz}), 1.45(\mathrm{~m}, 2 \mathrm{H}), 2.45(\mathrm{q}, 2 \mathrm{H}, J=7.4 \mathrm{~Hz}), 5.06\left(\mathrm{~d}, \mathrm{H}_{\text {cis }}, J=10.4 \mathrm{~Hz}\right), 5.57$ (d, $\left.\mathrm{H}_{\text {trans }}, J=16.6 \mathrm{~Hz}\right), 6.26$ (t, $1 \mathrm{H}, J=7.1 \mathrm{~Hz}, \mathrm{H}-4), 6.39(\mathrm{dd}, 1 \mathrm{H}, J=10.4,16.6 \mathrm{~Hz}, \mathrm{H}-2), 7.10-7.32(\mathrm{~m}, 5 \mathrm{H}) ;{ }^{13} \mathrm{C}$ NMR d (ppm) $14.0(\mathrm{Me}), 22.6(\mathrm{C}-6), 34.6$ (C-5), 117.3 (C-1), 125.9, 127.8, 129.1, 129.3, 131.3 (C-3), 131.6, 138.4 (C-2), 144.3 (C-4). Anal. Calcd for $\mathrm{C}_{13} \mathrm{H}_{16}$ Se: C, 62.15; H, 6.42. Found: C, 62.33; H, 6.26. (E)-isomer: oil, ${ }^{77} \mathrm{Se} \delta(\mathrm{ppm}) 375.8 ;{ }^{1} \mathrm{H}$ NMR $\delta(\mathrm{ppm}) 1.01(\mathrm{t}, 3 \mathrm{H}, J=7.4 \mathrm{~Hz}), 1.55$ $(\mathrm{m}, 2 \mathrm{H}), 2.45$ (q, 2H, J=7.4 Hz), 5.22 (d, $\left.\mathrm{H}_{\mathrm{cis}}, \mathrm{J}=10.3 \mathrm{~Hz}\right), 5.63$ (d, $\left.\mathrm{H}_{\text {trans }}, J=16.6 \mathrm{~Hz}\right), 6.03$ (t, 1H, J=7.1 Hz, H-4), 6.67 (dd, 
1H, $J=10.3,16.6 \mathrm{~Hz}, \mathrm{H}-2), 7.10-7.32$ (m, 5H); ${ }^{13} \mathrm{C}$ NMR d (ppm) 14.0 (Me), 22.7 (C-6), 34.4 (C-5), 120.0 (C-1), 125.9, $127.8,129.1,129.3,131.2(\mathrm{C}-3), 140.9(\mathrm{C}-2), 143.9$ (C-4).

3.1.2.3. 1-Phenyl-2-phenylselanylbut-1,3-diene 3c. Yield 67\%, Z/E 36/64. (Z)-isomer: oil, ${ }^{77} \mathrm{Se} \delta$ (ppm) 297.1 ; ${ }^{1} \mathrm{H}$ NMR d (ppm) $5.20\left(\mathrm{~d}, \mathrm{H}_{\text {cis }}, J=10.2 \mathrm{~Hz}\right), 5.70$ (d, H $\left.\mathrm{H}_{\text {trans }}, J=16.6 \mathrm{~Hz}\right), 6.51$ (ddd, $\left.1 \mathrm{H}, J=0.8,10.2,16.6 \mathrm{~Hz}, \mathrm{H}-3\right), 7.15$ (s, 1H, H-1), 7.25-7.40 (m, 8H), 7.60 (m, 2H); ${ }^{13} \mathrm{C}$ NMR $\delta(\mathrm{ppm}) 119.2(\mathrm{C}-4), 126.4,127.3,127.7,128.3,129.3,129.4,130.2,132.7$, 139.2 (C-3), 139.5, 140.9 (C-1). (E)-isomer: oil, ${ }^{77} \mathrm{Se} \delta$ (ppm) 409.2; ${ }^{1} \mathrm{H}$ NMR d (ppm) 5.34 (dd, $\left.\mathrm{H}_{\mathrm{cis}}, J=1.3,10.5 \mathrm{~Hz}\right), 5.80$ (d, $\left.\mathrm{H}_{\text {trans }}, J=16.6 \mathrm{~Hz}\right), 6.83$ (ddd, $\left.1 \mathrm{H}, J=1.0,10.5,16.6 \mathrm{~Hz}, \mathrm{H}-3\right), 7.05(\mathrm{~s}, 1 \mathrm{H}, \mathrm{H}-1), 7.25-7.40(\mathrm{~m}, 8 \mathrm{H}), 7.50(\mathrm{~m}, 2 \mathrm{H}) ;{ }^{13} \mathrm{C}$ NMR d (ppm) 121.4 (C-4), 126.4, 127.7, 128.1, 128.5, 129.4, 130.1, 130.6, 132.7, 133.2 (C-3), 137.5 (C-1). Anal. Calcd for $\mathrm{C}_{16} \mathrm{H}_{14} \mathrm{Se}: \mathrm{C}, 67.37 ; \mathrm{H}, 4.90$. Found: C, 66.97; H, 4.83 .

3.1.3. Preparation of selenides 4. The general procedure was used with the following modification. The reaction was carried out with ethyltriphenylphosphonium bromide. The selenide 4 was obtained after chromatography on silica gel (light petroleum) and rectification by Kugelrhor distillation.

3.1.3.1. 3-Phenylselanylhex-2,4-diene 4a. Yield 74\%. (2Z,4Z)-isomer: oil, ${ }^{77} \mathrm{Se} \delta(\mathrm{ppm}) 362.7 ;{ }^{1} \mathrm{H} \mathrm{NMR} \delta$ (ppm) $1.73(\mathrm{~d}, 3 \mathrm{H}$, $J=6.9 \mathrm{~Hz}, \mathrm{H}-6), 1.96$ (d, 3H, J=6.7 Hz, H-1), 5.40 (qd, J=6.9, $11.2 \mathrm{~Hz}, 1 \mathrm{H}, \mathrm{H}-5), 5.83$ (d, 1H, J=11.2 Hz, H-4), 5.97 (q, 1H, $J=6.7 \mathrm{~Hz}, \mathrm{H}-2), 7.10-7.25(\mathrm{~m}, 5 \mathrm{H}) ;{ }^{13} \mathrm{C}$ NMR $\delta(\mathrm{ppm}) 14.3,17.8,125.7,126.7,128.9,129.1,130.9,131.6,132.7,133.3$. Anal. Calcd for $\mathrm{C}_{12} \mathrm{H}_{14} \mathrm{Se}$ : C, 60.76; H, 5.95. Found: C, 60.68; H, 5.92. (2E,4Z)-isomer: oil, ${ }^{77} \mathrm{Se} \delta$ (ppm) $335.5 ;{ }^{1} \mathrm{H}$ NMR $\delta$ (ppm) 1.55 (dd, 3H, $J=1.5,6.9 \mathrm{~Hz}, \mathrm{H}-6), 1.67$ (dd, 3H, $J=1.3,6.9 \mathrm{~Hz}, \mathrm{H}-1), 5.51$ (m, 1H, H-5), 5.83 (d, 1H, $J=13.0 \mathrm{~Hz}, \mathrm{H}-4)$, 6.17 (m, 1H, H-2), 7.10-7.25 (m, 5H). (2E,4E)-isomer: oil, ${ }^{77} \mathrm{Se} \delta(\mathrm{ppm}) 399.8 ;{ }^{1} \mathrm{H}$ NMR $\delta$ (ppm) 1.78 (dd, 3H, $J=0.8,6.7$ Hz, H-6), 1.88 (d, 3H, J=7.7 Hz, H-1), 6.10 (m, 1H, H-5), 6.39 (d, 1H, J=15.1 Hz, H-4), 7.10-7.25 (m, 4H), 7.57-7.63 (m, $2 \mathrm{H}) ;{ }^{13} \mathrm{C}$ NMR $\delta$ (ppm) $15.3,18.4,126.1,126.3,128.9,129.3,131.0,132.0,133.3,134.7$. (2Z,4E)-isomer: oil, ${ }^{77} \mathrm{Se} \delta(\mathrm{ppm})$ 265.1; ${ }^{1} \mathrm{H}$ NMR $\delta$ (ppm) 1.72 (d, 3H, J=5.2 Hz, H-6), 1.97 (d, 3H, J=6.9 Hz, H-1), 6.05-6.16 (m, 2H, H-4, H-5), 6.18 (q, 1H, $J=6.9 \mathrm{~Hz}, \mathrm{H}-2), 7.10-7.25$ (m, 3H), 7.57-7.63 (m, 2H); ${ }^{13} \mathrm{C}$ NMR $\delta$ (ppm) 17.9, 18.3, 125.7, 127.8, 129.1, 129.7, 130.9, 131.6, 133.0, 135.5.

3.1.3.2. 4-Phenylselanyloct-2,4-diene 4b. Yield 69\%. (2Z,4Z)-isomer: oil, ${ }^{77} \mathrm{Se} \delta$ (ppm) 369.2; ${ }^{1} \mathrm{H} \mathrm{NMR} \delta(\mathrm{ppm}) 0.97(\mathrm{t}, 3 \mathrm{H}$, $J=7.4 \mathrm{~Hz}, \mathrm{H}-8), 1.51$ (m, 2H, H-7), 1.74 (dd, 3H, J=1.8, 6.9 Hz, H-1), 2.37 (m, 2H, H-6), 5.41 (m, 1H, H-2), 5.82 (d, 1H, $J=11.5 \mathrm{~Hz}, \mathrm{H}-3), 5.90$ (t, 1H, J=7.2 Hz, H-5), 7.10-7.30 (m, 3H), 7.35-7.42 (m, 2H); ${ }^{13} \mathrm{C}$ NMR $\delta$ (ppm) 14.0, 14.3 (C-1), 22.7, 34.2, 126.6 (C-2), 127.9, 128.9, 129.3, 131.0 (C-4), 131.6, 131.9 (C-3), 137.85 (C-5). Anal. Calcd for $\mathrm{C}_{14} \mathrm{H}_{18} \mathrm{Se}: \mathrm{C}$, 63.39; H, 6.84. Found: C, 63.13; H, 6.94. (2E,4Z)-isomer: oil, ${ }^{77} \mathrm{Se} \delta(\mathrm{ppm}) 273.3 ;{ }^{1} \mathrm{H}$ NMR d (ppm) 0.90 (t, $\left.3 \mathrm{H}, J=7.4 \mathrm{~Hz}\right)$, $1.43(\mathrm{~m}, 2 \mathrm{H}), 1.71(\mathrm{~d}, 3 \mathrm{H}, J=5.4 \mathrm{~Hz}), 2.40(\mathrm{~m}, 2 \mathrm{H}), 6.01-6.17(\mathrm{~m}, 3 \mathrm{H}), 7.10-7.30(\mathrm{~m}, 3 \mathrm{H}), 7.58-7.62(\mathrm{~m}, 2 \mathrm{H}) ;{ }^{13} \mathrm{C} \mathrm{NMR} \mathrm{d}$ (ppm) 14.0, 18.0 (C-1), 22.6, 34.4, 125.7 (C-3), 128.0, 129.3, 131.6, 133.0 (C-2), 141.1 (C-5). (2E,4E)-isomer: oil, ${ }^{77} \mathrm{Se} \delta$ (ppm) 400.8; ${ }^{1} \mathrm{H}$ NMR $\delta(\mathrm{ppm}) 0.95(\mathrm{t}, 3 \mathrm{H}, J=7.4 \mathrm{~Hz}), 1.45(\mathrm{~m}, 2 \mathrm{H}), 1.77$ (d, 3H, J=6.4 Hz), 2.29 (m, 2H), $6.02-6.18$ (m, $1 \mathrm{H}), 6.38(\mathrm{~d}, 1 \mathrm{H}, J=14.8 \mathrm{~Hz}), 7.15-7.30(\mathrm{~m}, 4 \mathrm{H}), 7.58-7.62(\mathrm{~m}, 2 \mathrm{H})$.

3.1.3.3. 1-Phenyl-2-phenylselanylpent-1,3-diene 4c. Yield 91\%. (1Z,3Z)-isomer: oil, ${ }^{77} \mathrm{Se} \delta$ (ppm) $411.0 ;{ }^{1} \mathrm{H}$ NMR $\delta$ (ppm) 1.77 (dd, 3H, J=1.5, $6.9 \mathrm{~Hz}), 5.40$ (m, 1H, H-4), 5.79 (d, 1H, J=11.3 Hz, H-3), 6.78 (s, 1H, H-1), 7.18-7.35 (m, 8H), 7.50$7.55(\mathrm{~m}, 2 \mathrm{H}) ;{ }^{13} \mathrm{C}$ NMR $\delta(\mathrm{ppm})$ 14.5, $127.6(\mathrm{C}-4), 127.8,128.3,128.4,128.9,129.2,129.3,129.9(\mathrm{C}-2), 132.2(\mathrm{C}-3), 132.9$ (C-1), 137.4. (1E,3Z)-isomer: oil, ${ }^{77} \mathrm{Se} \mathrm{d}(\mathrm{ppm}) 309.0 ;{ }^{1} \mathrm{H}$ NMR d (ppm) 1.38 (dd, 3H, J=1.5, 7.2 Hz), 5.43 (m, 1H, H-4), $6.04(\mathrm{~d}, 1 \mathrm{H}, \mathrm{J}=11.3 \mathrm{~Hz}, \mathrm{H}-3), 6.72$ (s, 1H, H-1), 7.18-7.35 (m, 8H), 7.50-7.55 (m, 2H). (1E,3E)-isomer: first eluted, $\mathrm{mp}=47$ C, ${ }^{77} \mathrm{Se} \mathrm{d}(\mathrm{ppm}) 418.5 ;{ }^{1} \mathrm{H}$ NMR d (ppm) $1.70\left(\mathrm{dd}, 3 \mathrm{H}, \mathrm{J}{ }^{1 / 40.8}, 6.7 \mathrm{~Hz}\right), 6.25(\mathrm{~m}, 1 \mathrm{H}, \mathrm{H}-4), 6.48(\mathrm{~d}, 1 \mathrm{H}, \mathrm{J} 1 / 414.6 \mathrm{~Hz}, \mathrm{H}-3)$, 6.77 (s, 1H, H-1), 7.25-7.36 (m, 8H), 7.49-7.54 (m, 2H); ${ }^{13} \mathrm{C}$ NMR d (ppm) 18.5 (C-5), 126.6, 127.2, 127.8 (C-3), 128.4, 129.2, 129.4, 131.0, 132.3 (C-2), 132.7, 133.9 (C-4), 134.6 (C-1), 137.4. Anal. Calcd for $\mathrm{C}_{17} \mathrm{H}_{16} \mathrm{Se}$ : C, 68.23; H, 5.39. Found: C, 68.11; H, 5.35.

3.1.4. Preparation of dienes 5. A solution of $n$-BuLi in hexanes (1.6 M, $3.45 \mathrm{ml}, 5.5 \mathrm{mmol})$ was slowly added, under argon, to benzyltriphenylphosphonium chloride $(2.13 \mathrm{~g}, 5.5 \mathrm{mmol})$ in anhydrous THF $(50 \mathrm{ml})$. After stirring for $1 \mathrm{~h}$ at room temperature, $\alpha$-phenylselanyl enal $2(5 \mathrm{mmol})$ in THF $(5 \mathrm{ml})$ was added dropwise. The mixture was stirred for $3 \mathrm{~h}$ at room temperature, quenched with saturated aqueous $\mathrm{NH}_{4} \mathrm{Cl}$, and extracted with diethylether. The organic extracts were dried over magnesium sulfate and concentrated under vacuum. The residue was purified by chromatography on silica gel (light petroleum $/ \mathrm{CH}_{2} \mathrm{Cl}_{2}: 80 / 20$ ).

3.1.4.1. 1-Phenyl-3-phenylselanylpent-1,3-diene 5a. Yield 88\%. (1Z,3Z)-isomer: oil, ${ }^{77} \mathrm{Se} \delta$ (ppm) 368.6; ${ }^{1} \mathrm{H}$ NMR $\delta$ (ppm) 1.79 (dd, 3H, $J=1.6,6.9 \mathrm{~Hz}), 5.93-6.04$ (m, 2H, H-1, H-4), 6.14 (d, $1 \mathrm{H}, J=12.0 \mathrm{~Hz}, \mathrm{H}-2), 7.10-7.60$ (m, $10 \mathrm{H}) ;{ }^{13} \mathrm{C}$ NMR $\delta$ (ppm) 17.5, 126.8, 128.6, 128.7, 129.3, 129.8, 131.1 (C-2), 131.8 (C-1), 138.1 (C-3), 138.9 (C-4). (1E,3Z)-isomer: oil, ${ }^{77} \mathrm{Se} \delta$ 
(ppm) 261.7; ${ }^{1} \mathrm{H}$ NMR $\delta$ (ppm) 2.07 (d, 3H, $\left.J=6.9 \mathrm{~Hz}\right), 6.45$ (q, 1H, J=6.9 Hz, H-4), 6.85 (d, 1H, J=15.5 Hz, H-1), 7.01 (d, $1 \mathrm{H}, J=15.5 \mathrm{~Hz}, \mathrm{H}-2), 7.10-7.45(\mathrm{~m}, 10 \mathrm{H}) ;{ }^{13} \mathrm{C}$ NMR $\delta(\mathrm{ppm}) 18.7,126.8,128.6,128.7,129.3,129.8,131.1(\mathrm{C}-2), 131.6(\mathrm{C}-$ 1), 137.4 (C-3), 139.0 (C-4). Anal. Calcd for $\mathrm{C}_{17} \mathrm{H}_{16} \mathrm{Se}: \mathrm{C}, 68.23$; H, 5.39. Found: C, 68.54; H, 5.62. (1E,3E)-isomer: oil, ${ }^{77} \mathrm{Se}$ (ppm) 396.6; ${ }^{1} \mathrm{H}$ NMR $\delta(\mathrm{ppm}) 2.02(\mathrm{~d}, 3 \mathrm{H}, J=7.3 \mathrm{~Hz}), 6.38(\mathrm{q}, 1 \mathrm{H}, J=7.3 \mathrm{~Hz}, \mathrm{H}-4), 7.03$ (d, 1H, $\left.J=15.5 \mathrm{~Hz}, \mathrm{H}-1\right), 7.13$ (d, $1 \mathrm{H}, J=15.5 \mathrm{~Hz}, \mathrm{H}-2), 7.15-7.60(\mathrm{~m}, 10 \mathrm{H}) ;{ }^{13} \mathrm{C}$ NMR $\delta(\mathrm{ppm}) 15.7,126.0,128.7,129.3,129.8,130.9$ (C-2), 131.6, 132.8 (C1), 137.2 (C-3), $138.2(\mathrm{C}-4)$.

3.1.4.2. 1-Phenyl-3-phenylselanylhept-1,3-diene 5b. Yield 82\%. (1Z,3Z)-isomer: ${ }^{77} \mathrm{Se} \delta$ (ppm) $374.9 ;{ }^{1} \mathrm{H}$ NMR $\delta$ (ppm) 0.87 (t, 3H, J=7.4 Hz), $1.37(\mathrm{~m}, 2 \mathrm{H}), 2.24(\mathrm{~m}, 2 \mathrm{H}), 5.96(\mathrm{t}, 1 \mathrm{H}, J=7.2 \mathrm{~Hz}, \mathrm{H}-4), 5.98$ (d, 1H, J=12.0 Hz, H-1), 6.16 (d, $1 \mathrm{H}, J=12.0$ $\mathrm{Hz}, \mathrm{H}-2), 7.10-7.60$ (m, 10H); ${ }^{13} \mathrm{C}$ NMR $\delta$ (ppm) 14.0, 21.9, 33.9, 124.0, 126.7, 128.7, 129.3, 129.8, 130.7, 131.1, 132.2, $137.3(\mathrm{C}-3), 144.5$ (C-4). (1E,3Z)-isomer: ${ }^{77} \mathrm{Se} \delta$ (ppm) 269.8; ${ }^{1} \mathrm{H}$ NMR (ppm) 0.91 (t, 3H, J=7.4 Hz), $1.44(\mathrm{~m}, 2 \mathrm{H}), 2.47(\mathrm{~m}$, 2H), 6.35 (t, 1H, J=7.4 Hz, H-4), $6.82(\mathrm{~d}, 1 \mathrm{H}, \mathrm{J}=15.4 \mathrm{~Hz}, \mathrm{H}-1), 6.97$ (d, 1H, J=15.4 Hz, H-2), 7.10-7.60 (m, 10H); ${ }^{13} \mathrm{C}$ NMR $\delta(\mathrm{ppm}) 14.1,22.6,34.8,124.0,126.7,128.7,129.3,129.8,130.7$ (C-2), 131.1, $132.2(\mathrm{C}-1), 137.4$ (C-3), 144.6 (C-4). Anal. Calcd for $\mathrm{C}_{19} \mathrm{H}_{20} \mathrm{Se}$ : $\mathrm{C}, 69.72 ; \mathrm{H}, 6.16$. Found: $\mathrm{C}, 69.54 ; \mathrm{H}, 5.92$. (1E,3E)-isomer: ${ }^{77} \mathrm{Se} \delta(\mathrm{ppm}) 397.9 ;{ }^{1} \mathrm{H}$ NMR $\delta(\mathrm{ppm}) 0.96$ (t, 3H, J=7.4 Hz), $1.51(\mathrm{~m}, 2 \mathrm{H}), 2.39(\mathrm{~m}, 2 \mathrm{H}), 6.30$ (t, 1H, J=7.4 Hz, H-4), 7.05 (d, 1H, J=15.4 Hz, H-1), 7.10-7.60 (m, $11 \mathrm{H}) ;{ }^{13} \mathrm{C}$ NMR d (ppm) 14.0, 22.6, 31.9, 124.0, 126.7, 128.7, 129.3, 129.8, 130.8 (C-2), 131.1, 132.2 (C-1), 137.7 (C-3), $144.3(\mathrm{C}-4)$.

3.1.4.3. 1,4-Diphenyl-2-phenylselanylbut-1,3-diene 5c. ${ }^{8}$ Yield 94\%. (1Z,3Z)-isomer: ${ }^{77} \mathrm{Se} \delta$ (ppm) 415.4; ${ }^{1} \mathrm{H}$ NMR $\delta$ (ppm) $5.98(\mathrm{dd}, 1 \mathrm{H}, J=1.5,12.0 \mathrm{~Hz}, \mathrm{H}-4), 6.18(\mathrm{~d}, 1 \mathrm{H}, \mathrm{J}=12.0 \mathrm{~Hz}, \mathrm{H}-3), 6.92(\mathrm{~d}, 1 \mathrm{H}, J=1.5 \mathrm{~Hz}, \mathrm{H}-1), 7.15-7.60$ (m, 15H); ${ }^{13} \mathrm{C}$ NMR $\delta(\mathrm{ppm}) 126.9,127.2,127.7,128.1,128.5,128.7,128.8,129.4,129.5,130.5$ (C-3), 130.9 (C-1), $131.8(\mathrm{C}-4)$, 133.7, 134.0, 136.0, 137.7 (C-2). (1Z,3E)-isomer: ${ }^{12}{ }^{77} \mathrm{Se} \delta(\mathrm{ppm}) 304.0 ;{ }^{1} \mathrm{H}$ NMR $\delta(\mathrm{ppm}) 7.00(\mathrm{~d}, 1 \mathrm{H}, J=15.4 \mathrm{~Hz}, \mathrm{H}-4)$, 7.15-7.60 (m, 17H).

3.1.5. Preparation of dienic methylketones 6. A solution of $\alpha$-phenylselanyl enal $2(5 \mathrm{mmol})$ and 1-triphenylphosphoranylidene propanone $(1.75 \mathrm{~g}, 5.5 \mathrm{mmol})$ in chloroform $(30 \mathrm{ml})$ was stirred at room temperature for $48 \mathrm{~h}(\mathbf{6 a})$ or $60 \mathrm{~h}(\mathbf{6 c})$. After concentration under reduced pressure, the residue was purified by silica gel chromatography (light petroleum $/ \mathrm{CH}_{2} \mathrm{Cl}_{2}$ : 70/30).

3.1.5.1. 5-Phenylselanylhepta-3,5-dien-2-one 6a. Yield 67\%. (3E,5Z)-isomer: ${ }^{77} \mathrm{Se} \delta$ (ppm) 267.4; ${ }^{1} \mathrm{H}$ NMR $\delta(\mathrm{ppm}) 2.11(\mathrm{~d}$, $3 \mathrm{H}, J=6.9 \mathrm{~Hz}, \mathrm{H}-7), 2.23$ (s, 3H, H-1), 6.58 (d, 1H, $J=15.2 \mathrm{~Hz}, \mathrm{H}-3), 6.75$ (q, 1H, $J=6.9 \mathrm{~Hz}, \mathrm{H}-6), 7.10-7.30(\mathrm{~m}, 6 \mathrm{H}) ;{ }^{13} \mathrm{C}$ NMR $\delta$ (ppm) 19.2 (C-7), 28.4 (C-1), 126.8, 129.2, 129.8 (C-3), 131.0, 134.4, 136.9 (C-5), 145.3 (C-4), 148.0 (C-6), 198.3 (C-2). Anal. Calcd for $\mathrm{C}_{13} \mathrm{H}_{14} \mathrm{OSe}$ : C, 58.87; H, 5.32. Found: C, 58.51; H, 5.15. (3E,5E)-isomer: ${ }^{77} \mathrm{Se} \delta(\mathrm{ppm}) 401.6 ;{ }^{1} \mathrm{H}$ NMR d (ppm) 2.04 (d, 3H, J=7.4 Hz, H-7), 2.25 (s, 3H, H-1), 6.59 (d, 1H, J=15.2 Hz, H-3), 6.71 (q, 1H, J=7.4 Hz, H-6), 7.10-7.30 (m, 5H), $7.56(\mathrm{~d}, 1 \mathrm{H}, J=15.2 \mathrm{~Hz}, \mathrm{H}-4)$.

3.1.5.2. 5-Phenylselanylnona-3,5-dien-2-one 6b. Yield 77\%. (3E,5Z)-isomer: ${ }^{77} \mathrm{Se} \delta(\mathrm{ppm}) 275.6 ;{ }^{1} \mathrm{H}$ NMR (ppm) $0.93(\mathrm{t}$, $3 \mathrm{H}, J=7.4 \mathrm{~Hz}, \mathrm{H}-9), 1.49$ (m, 2H, H-8), 2.23 (s, 3H, H-1), 2.52 (m, 2H, H-7), 6.56 (d, 1H, J=15.1 Hz, H-3), 6.67 (t, $1 \mathrm{H}$, $\mathrm{J}=7.2 \mathrm{~Hz}, \mathrm{H}-6), 7.10-7.35$ (m, 6H, H-4, Ph); ${ }^{13} \mathrm{C}$ NMR d (ppm) 13.9 (C-9), 22.1 (C-8), 28.5 (C-1), 35.3 (C-7), 126.4, 129.5, 129.9 (C-3), 136.8 (C-5), 145.5 (C-4), 153.5 (C-6), 198.6 (C-2). Anal. Calcd for $\mathrm{C}_{15} \mathrm{H}_{18} \mathrm{OSe}$ : C, 61.43; H, 6.19. Found: C, 61.71; H, 6.26. (3E,5E)-isomer: ${ }^{77} \mathrm{Se} \delta$ (ppm) 403.7; ${ }^{1} \mathrm{H}$ NMR (ppm) 0.96 (t, 3H, $J=7.4 \mathrm{~Hz}, \mathrm{H}-9$ ), 1.48 (m, 2H, H-8), 2.25 (s, 3H, H-1), 2.42 (m, 2H, H-7), 6.56 (d, 1H, J=15.1 Hz, H-3), 6.62 (t, 1H, J=7.2 Hz, H-6), 7.10-7.35 (m, 5H, Ph), 7.54 (d, $1 \mathrm{H}, J=15.1 \mathrm{~Hz}, \mathrm{H}-4)$.

3.1.5.3. 6-Phenyl-5-phenylselanylhexa-3,5-dien-2-one 6c. Yield $88 \%$. (3E,5E)-isomer (cyclohexane/dichloromethane:85/15): ${ }^{77} \mathrm{Se} \delta(\mathrm{ppm}) 423.2 ;{ }^{1} \mathrm{H}$ NMR $\delta(\mathrm{ppm}) 2.21(\mathrm{~s}, 3 \mathrm{H}), 6.70(\mathrm{~d}, 1 \mathrm{H}, J=15.4 \mathrm{~Hz}, \mathrm{H}-3), 7.15-7.50(\mathrm{~m}, 11 \mathrm{H}), 7.61$ (dd, $1 \mathrm{H}, J=0.9,15.4 \mathrm{~Hz}, \mathrm{H}-4) ;{ }^{13} \mathrm{C}$ NMR d (ppm) 27.0 (C-1), 127.7 (C-3), 128.0, 128.2, 128.8, 129.4, 130.4, 131.1, 132.6, 134.4, 136.4 (C-5), 139.5 (C-6), 144.8 (C-4), 198.4 (C-2). (3E,5Z)-isomer (cyclohexane/ dichloromethane: 75/25): mp 45 C, ${ }^{77} \mathrm{Se} \delta(\mathrm{ppm}) 308.5 ;{ }^{1} \mathrm{H}$ NMR $\delta(\mathrm{ppm}) 2.23(\mathrm{~s}, 3 \mathrm{H}), 6.64(\mathrm{~d}, 1 \mathrm{H}, J=15.4 \mathrm{~Hz}, \mathrm{H}-3), 7.10-7.30$ (m, 9H), 7.55 (s, 1H, H-6), 7.67-7.71 (m, 2H); ${ }^{13} \mathrm{C}$ NMR $\delta$ (ppm) $28.5(\mathrm{C}-1), 126.8$ (C-3), 127.9, 128.0, 128.2, 129.4, 130.4, 131.1, 132.6, 134.4, 136.0 (C-5), 146.4 (C-6), 146.8 (C-4), 198.4 (C-2). Anal. Calcd for $\mathrm{C}_{18} \mathrm{H}_{16} \mathrm{OSe}$ : C, 66.06; H, 4.93. Found: C, 65.90; H, 4.86.

3.1.6. Preparation of penta-2,4-dienenitriles 7. A solution of $n$-BuLi in hexanes (1.6 M, $3.45 \mathrm{ml}, 5.5 \mathrm{mmol})$ was slowly added, under argon, to cyanomethyldiethylphosphonoacetate $(0.97 \mathrm{~g}, 5.5 \mathrm{mmol})$ in anhydrous THF $(50 \mathrm{ml})$ at $0 \mathrm{C}$. After stirring for $0.5 \mathrm{~h}$ at room temperature, the solution was cooled to $78^{\circ} \mathrm{C}$. Then, $\alpha$-phenylselanyl enal 2 ( $5 \mathrm{mmol}$ ) in THF (5 $\mathrm{ml}$ ) was added dropwise at this temperature. The mixture was stirred for $2 \mathrm{~h}$ at $78 \mathrm{C}$ for $\mathbf{7 a}$ and $\mathbf{7 b}$ or $12 \mathrm{~h}$ for $\mathbf{7 c}$, quenched 
with saturated aqueous $\mathrm{NH}_{4} \mathrm{Cl}$, and extracted with diethylether. The organic extracts were dried over magnesium sulfate and concentrated under vacuum. The residue was purified by chromatography on silica gel (light petroleum/ $\mathrm{CH}_{2} \mathrm{Cl}_{2}: 70 / 30$ ).

3.1.6.1. 4-Phenylselanylhex-2,4-dienenitrile 7a. Yield 61\%. (2E,4Z)-isomer: ${ }^{77} \mathrm{Se} \delta$ (ppm) $252.7 ;{ }^{1} \mathrm{H}$ NMR (ppm) $2.13(\mathrm{~d}$, $3 \mathrm{H}, \mathrm{J}=6.9 \mathrm{~Hz}, \mathrm{H}-6), 5.84$ (d, 1H, J=15.6 Hz, H-2), 6.69 (q, 1H, J=6.9 Hz, H-5), 7.09 (d, 1H, J=15.7 Hz, H-3), 7.17-7.25 (m, $5 \mathrm{H}) ;{ }^{13} \mathrm{C}$ NMR $\delta$ (ppm) 19.3, 99.7 (C-2), 118.3 (C-1), 126.7, 129.4, 129.9, 130.6, 132.0, 148.6 (C-5), 152.1 (C-3). Anal. Calcd for $\mathrm{C}_{12} \mathrm{H}_{11} \mathrm{NSe}$ : C, 58.07; H, 4.47. Found: C, 58.13; H, 4.58. (2E,4E)-isomer: ${ }^{77} \mathrm{Se} \delta$ (ppm) $385.8 ;{ }^{1} \mathrm{H}$ NMR $\delta$ (ppm) 2.04 (d, 3H, $J=7.4 \mathrm{~Hz}, \mathrm{H}-6), 5.89$ (d, 1H, $J=15.6 \mathrm{~Hz}, \mathrm{H}-2), 6.77$ (q, 1H, $J=7.3 \mathrm{~Hz}, \mathrm{H}-5$ ), 7.42 (d, 1H, $J=15.6 \mathrm{~Hz}, \mathrm{H}-3$ ), $7.17-$ 7.25 (m, 5H); ${ }^{13} \mathrm{C}$ NMR d (ppm) 16.1, 102.4, 118.3, 126.7, 129.3, 129.8, 132.0, 144.4, 151.8. (2Z,4Z)-isomer: ${ }^{77} \mathrm{Se} \delta(\mathrm{ppm})$ 348.5; ${ }^{1} \mathrm{H}$ NMR $\delta(\mathrm{ppm}) 2.10$ (dd, 3H, J=0.9, $\left.6.9 \mathrm{~Hz}, \mathrm{H}-6\right), 5.09$ (d, 1H, J=11.4 Hz, H-2), 6.69 (dd, 1H, J=0.9, $\left.11.4 \mathrm{~Hz}, \mathrm{H}-3\right)$, $6.76(\mathrm{qd}, 1 \mathrm{H}, \mathrm{J}=0.9,7.3 \mathrm{~Hz}, \mathrm{H}-5), 7.19-7.25(\mathrm{~m}, 3 \mathrm{H}), 7.38-7.45(\mathrm{~m}, 2 \mathrm{H}) ;{ }^{13} \mathrm{C}$ NMR $\delta(\mathrm{ppm}) 18.4,96.7(\mathrm{C}-2), 116.6(\mathrm{C}-1)$, 126.4, 127.4, 129.3, 129.7, 140.4, 147.5. (2Z,4E)-isomer: ${ }^{77} \mathrm{Se} \delta(\mathrm{ppm}) 304.5 ;{ }^{1} \mathrm{H}$ NMR $\delta(\mathrm{ppm}) 1.94(\mathrm{dd}, 3 \mathrm{H}, J=1.0,7.2 \mathrm{~Hz}$, H-6), 5.25 (d, 1H, J=11.2 Hz, H-2), 6.70 (dd, 1H, J=0.9, 11.2 Hz, H-3), 6.55 (qd, 1H, J=1.0, 7.2 Hz, H-5), 7.19-7.25 (m, $3 \mathrm{H}), 7.38-7.45(\mathrm{~m}, 2 \mathrm{H})$.

3.1.6.2. 4-Phenylselanyloct-2,4-dienenitrile 7b. Yield 82\%. (2E,4Z)-isomer: ${ }^{77} \mathrm{Se} \delta(\mathrm{ppm}) 260.7 ;{ }^{1} \mathrm{H}$ NMR d (ppm) $0.95(\mathrm{t}$, $3 \mathrm{H}, \mathrm{J}=7.0 \mathrm{~Hz}, \mathrm{H}-8), 1.50$ (m, 2H, H-7), 2.54 (q, 2H, J=7.0 Hz, H-6), 5.83 (d, 1H, J=15.6 Hz, H-2), 6.62 (t, 1H, J=7.4 Hz, H5), $7.10(\mathrm{~d}, 1 \mathrm{H}, \mathrm{J}=15.6 \mathrm{~Hz}, \mathrm{H}-3), 7.12-7.40(\mathrm{~m}, 5 \mathrm{H}) ;{ }^{13} \mathrm{C}$ NMR d (ppm) 13.9, 21.9, 35.2, 99.8 (C-2), 118.2 (C-1), 126.3, 126.7, 128.8 (C-4), 129.2, 129.7, 152.0 (C-3), 153.7 (C-5). Anal. Calcd for $\mathrm{C}_{14} \mathrm{H}_{15} \mathrm{NSe}$ : C, 60.87; H, 5.47; N, 5.07. Found: C, 60.63; H, 5.28; N, 4.78. (2E,4E)-isomer: ${ }^{77} \mathrm{Se} \delta(\mathrm{ppm}) 387.1 ;{ }^{1} \mathrm{H}$ NMR $\delta(\mathrm{ppm}) 0.98(\mathrm{t}, 3 \mathrm{H}, \mathrm{J}=7.0 \mathrm{~Hz}, \mathrm{H}-8), 1.52(\mathrm{~m}, 2 \mathrm{H}, \mathrm{H}-$ 7), 2.52 (q, 2H, J=7.0 Hz, H-6), $5.90(\mathrm{~d}, 1 \mathrm{H}, \mathrm{J}=15.6 \mathrm{~Hz}, \mathrm{H}-2), 6.69(\mathrm{t}, 1 \mathrm{H}, \mathrm{J}=7.1 \mathrm{~Hz}, \mathrm{H}-5), 7.12-7.50(\mathrm{~m}, 6 \mathrm{H}) ;{ }^{13} \mathrm{C}$ NMR $\delta$ (ppm) 12.7, 22.3, 32.0, 102.4 (C-2), 116.6 (C-1), 125.6, 127.0, 128.8 (C-4), 129.2, 129.7, 144.7 (C-5), 153.8 (C-3). (2Z,4Z)isomer: ${ }^{77} \mathrm{Se} \delta(\mathrm{ppm}) 357.3 ;{ }^{1} \mathrm{H}$ NMR $\delta(\mathrm{ppm}) 0.97(\mathrm{t}, 3 \mathrm{H}, \mathrm{J}=7.0 \mathrm{~Hz}, \mathrm{H}-8), 1.50(\mathrm{~m}, 2 \mathrm{H}, \mathrm{H}-7), 2.39(\mathrm{q}, 2 \mathrm{H}, \mathrm{J}=7.0 \mathrm{~Hz}, \mathrm{H}-6)$, 5.09 (d, 1H, J=11.5 Hz, H-2), 6.65-6.73 (m, 2H, H-3, H-5), 7.12-7.40 (m, 5H); ${ }^{13} \mathrm{C}$ NMR d (ppm) 13.7, 22.0, 34.3, 96.9 (C2), 116.6 (C-1), 125.6, 127.2, 128.8 (C-4), 129.2, 129.7, 145.1 (C-5), 151.1 (C-3).

3.1.6.3. 5-Phenyl-4-phenylselanylpent-2,4-dienenitrile 7c. Yield 83\%. (2E,4Z)-isomer (cyclohexane/dichloro-methane: 80/20): mp $92 \mathrm{C},{ }^{77} \mathrm{Se} \delta(\mathrm{ppm}) 288.2 ;{ }^{1} \mathrm{H}$ NMR $\delta$ (ppm) 5.94 (d, 1H, J=15.6 Hz, H-2), 7.15-7.35 (m, 9H), 7.47 (s, 1H, H-5), 7.68-7.72 (m, 2H); ${ }^{13} \mathrm{C}$ NMR d (ppm) 101.3 (C-2), $118.4(\mathrm{C}-1), 126.9,127.1,128.4,129.7,130.0,130.2,130.6,135.3(\mathrm{C}-4)$, 147.6 (C-5), 153.0 (C-3). Anal. Calcd for $\mathrm{C}_{17} \mathrm{H}_{13} \mathrm{NSe}$ C, 65.81; H, 4.22; N, 4.51. Found: C, 65.87; H, 3.98; N, 4.38. (2E,4E)isomer (cyclohexane/dichloromethane: 90/10): ${ }^{77} \mathrm{Se} \delta$ (ppm) 408.6; ${ }^{1} \mathrm{H}$ NMR $\delta$ (ppm) 5.99 (d, 1H, J=15.8 Hz, H-2), 7.17$7.50(\mathrm{~m}, 11 \mathrm{H}), 7.55(\mathrm{~s}, 1 \mathrm{H}, \mathrm{H}-5) ;{ }^{13} \mathrm{C}$ NMR $\left.\delta(\mathrm{ppm}) 103.6(\mathrm{C}-2), 117.0(\mathrm{C}-1), 127.6,128.3,128.9,129.4, \mathrm{H}-2\right), 6.99(\mathrm{dd}, 1 \mathrm{H}$, $\mathrm{J} 1 / 41.3,11.8 \mathrm{~Hz}, \mathrm{H}-3), 7.15-7.35$ (m, 9H), 7.55-7.60 (m, 2H); ${ }^{13} \mathrm{C}$ NMR d (ppm) 101.1 (C-2), 116.1 (C-1), 126.6, 128.2, 128.6, 128.9, 129.1, 129.1, 129.6, 129.8, 130.0, 131.6, 135.4 (C-4), 146.2 (C-5), 152.8 (C-3). (2Z,4Z)-isomer (cyclohexane/dichloromethane: 85/15): ${ }^{77} \mathrm{Se} \delta(\mathrm{ppm}) 398.4 ;{ }^{1} \mathrm{H}$ NMR $\delta(\mathrm{ppm}) 5.14$ (d, 1H, J=11.3 Hz, H-2), $6.75(\mathrm{dd}, 1 \mathrm{H}$, $\mathrm{J}=1.0,11.3 \mathrm{~Hz}, \mathrm{H}-3), 7.15-7.35$ (m, 9H), 7.55-7.60 (m, 2H); ${ }^{13} \mathrm{C}$ NMR $\delta(\mathrm{ppm}) 97.7$ (C-2), 116.6 (C-1), 126.6, 128.2, 128.6, 128.9, 129.1, 129.1, 129.3, 133.7, 135.8 (C-4), 138.6 (C-5), 151.8 (C-3), 129.3, 133.7, 136.0 (C-4), 142.4 (C-5), 146.9 (C-3).

3.1.7. Preparation of dienic esters 8. A solution of $n$-BuLi in hexanes (1.6 M, $3.45 \mathrm{ml}, 5.5 \mathrm{mmol})$ was slowly added, under argon, to triethylphosphonoacetate $(1.2 \mathrm{~g}, 5.5 \mathrm{mmol})$ in anhydrous THF $(50 \mathrm{ml})$ at $0 \mathrm{C}$. After stirring for $0.5 \mathrm{~h}$ at room temperature, $\alpha$-phenylselanyl enal $2(5 \mathrm{mmol})$ in THF $(5 \mathrm{ml})$ was added dropwise. The mixture was stirred for $3 \mathrm{~h}$ at room temperature, quenched with saturated aqueous $\mathrm{NH}_{4} \mathrm{Cl}$, and extracted with diethylether. The organic extracts were dried over magnesium sulfate and concentrated under vacuum. The residue was purified by chromatography on silica gel (light petroleum/ $\mathrm{CH}_{2} \mathrm{Cl}_{2}: 80 / 20$ ).

3.1.7.1. Ethyl 4-phenylselanylhex-2,4-dienoate 8a ${ }^{8}$ Yield 72\%. (2E,4Z)-isomer: ${ }^{77} \mathrm{Se} \delta(\mathrm{ppm}) 263.6 ;{ }^{1} \mathrm{H}$ NMR (ppm) 1.27 $(\mathrm{t}, 3 \mathrm{H}, \mathrm{J}=7.1 \mathrm{~Hz}), 2.09(\mathrm{~d}, 3 \mathrm{H}, J=6.9 \mathrm{~Hz}), 4.17(\mathrm{q}, 2 \mathrm{H}, J=7.1 \mathrm{~Hz}), 6.30(\mathrm{dd}, 1 \mathrm{H}, J=0.6,15.1 \mathrm{~Hz}, \mathrm{H}-2), 6.71(\mathrm{q}, 1 \mathrm{H}, J=6.9 \mathrm{~Hz}$, H-5), 7.12-7.32 (m, 5H), 7.41 (d, 1H, J1/415.1 Hz, H-3); ${ }^{13} \mathrm{C}$ NMR d (ppm) 14.4, 19.2, 60.5, 121.6 (C-2), 126.3, 129.4, 129.8, 130.4 (C-4), 134.3, 146.9 (C-3), 147.5 (C-5), $167.4(\mathrm{C}-1)$. (2E,4E)-isomer: ${ }^{77} \mathrm{Se} \delta(\mathrm{ppm}) 399.6 ;{ }^{1} \mathrm{H}$ NMR $\delta(\mathrm{ppm}) 1.26(\mathrm{t}, 3 \mathrm{H}$, $\mathrm{J}=7.1 \mathrm{~Hz}$ ), 2.04 (d, 3H, J=7.4 Hz), 4.17 (q, 2H, J=7.1 Hz), 6.33 (d, 1H, J=14.8 Hz, H-2), 6.65 (q, 1H, J=7.1 Hz, H-5), 7.12$7.30(\mathrm{~m}, 5 \mathrm{H}), 7.72(\mathrm{~d}, 1 \mathrm{H}, \mathrm{J}=14.8 \mathrm{~Hz}, \mathrm{H}-3) ;{ }^{13} \mathrm{C}$ NMR $\delta(\mathrm{ppm}) 13.9,19.3,60.4,122.3(\mathrm{C}-2), 126.8,129.3,129.8,130.4(\mathrm{C}-$ 4), 134.4, 138.9 (C-3), 147.4 (C-5), $167.3(\mathrm{C}-1)$.

3.1.7.2. Ethyl 4-phenylselanyloct-2,4-dienoate $8 \mathbf{8 b} .{ }^{8}$ Yield 71\%. (2E,4Z)-isomer: ${ }^{77} \mathrm{Se} \delta(\mathrm{ppm}) 271.5$; ${ }^{1} \mathrm{H}$ NMR d (ppm) $0.92(\mathrm{t}, 3 \mathrm{H}, \mathrm{J}=7.0 \mathrm{~Hz}), 1.26(\mathrm{t}, 3 \mathrm{H}, \mathrm{J}=7.1 \mathrm{~Hz}), 1.48(\mathrm{~m}, 2 \mathrm{H}), 2.51(\mathrm{q}, 2 \mathrm{H}, \mathrm{J}=7.0 \mathrm{~Hz}), 4.17(\mathrm{q}, 2 \mathrm{H}, \mathrm{J}=7.1 \mathrm{~Hz}), 6.28(\mathrm{~d}, 1 \mathrm{H}$, $\mathrm{J}=15.1 \mathrm{~Hz}, \mathrm{H}-2), 6.63(\mathrm{t}, 1 \mathrm{H}, \mathrm{J}=7.1 \mathrm{~Hz}, \mathrm{H}-5), 7.12-7.30(\mathrm{~m}, 5 \mathrm{H}), 7.40$ (d, 1H, J=15.1 Hz, H-3); ${ }^{13} \mathrm{C}$ NMR d (ppm) 14.0, 14.4, 22.2, 35.2, 60.5, 121.8 (C-2), 126.3, 129.4, 129.8, 130.8, 131.0 (C-4), 150.0 (C-3), 152.9 (C-5), 167.4 (C-1). (2E,4E)- 
isomer: ${ }^{77} \mathrm{Se} \mathrm{d}(\mathrm{ppm}) 401.1 ;{ }^{1} \mathrm{H}$ NMR $\delta(\mathrm{ppm}) 0.96(\mathrm{t}, 3 \mathrm{H}, \mathrm{J}=7.0 \mathrm{~Hz}), 1.27(\mathrm{t}, 3 \mathrm{H}, \mathrm{J}=7.1 \mathrm{~Hz}), 1.51(\mathrm{~m}, 2 \mathrm{H}), 2.42(\mathrm{q}, 2 \mathrm{H}$, $\mathrm{J}=7.0 \mathrm{~Hz}), 4.17(\mathrm{q}, 2 \mathrm{H}, \mathrm{J}=7.1 \mathrm{~Hz}), 6.33(\mathrm{~d}, 1 \mathrm{H}, \mathrm{J}=15.1 \mathrm{~Hz}, \mathrm{H}-2), 6.59(\mathrm{t}, 1 \mathrm{H}, \mathrm{J}=7.1 \mathrm{~Hz}, \mathrm{H}-5), 7.12-7.30$ (m, 5H), $7.70(\mathrm{~d}, 1 \mathrm{H}$, $\mathrm{J}=15.1 \mathrm{~Hz}, \mathrm{H}-3) ;{ }^{13} \mathrm{C}$ NMR d (ppm) 13.8, 14.4, 22.5, 32.1, 60.6, 124.0 (C-2), 126.7, 129.0, 129.3, 129.8, 131.1 (C-4), 139.3 (C-3), $152.0(\mathrm{C}-5), 167.4(\mathrm{C}-1)$.

3.1.7.3. Ethyl 5-Phenyl-4-phenylselanylpent-2,4-dien-oate $8 \mathbf{c}^{8}{ }^{8}$ Yield $84 \%$. (2E,4Z)-isomer: $54 \%, \mathrm{mp}=55 \mathrm{C}$; ${ }^{77} \mathrm{Se} \delta(\mathrm{ppm})$ 301.1; ${ }^{1} \mathrm{H}$ NMR $\delta(\mathrm{ppm}) 1.28(\mathrm{t}, 3 \mathrm{H}, \mathrm{J}=7.2 \mathrm{~Hz}), 4.16(\mathrm{q}, 2 \mathrm{H}, \mathrm{J}=7.2 \mathrm{~Hz}), 6.38(\mathrm{~d}, 1 \mathrm{H}, \mathrm{J}=15.1 \mathrm{~Hz}, \mathrm{H}-2), 7.16-7.40(\mathrm{~m}, 8 \mathrm{H})$, 7.52 (s, 1H, H-5), 7.55 (d, 1H, J1/415.1 Hz, H-3), 7.67-7.73 (m, 2H); ${ }^{13} \mathrm{C}$ NMR d (ppm) 14.4, 60.6, 123.1 (C-2), 126.6, 127.5 (C-4), 128.3, 129.3, 129.4, 130.1, 130.4, 130.7, 136.0, 146.7 (C-3), 147.8 (C-5), 167.1 (C-1). (2E,4E)-isomer: ${ }^{77} \mathrm{Se}(\mathrm{ppm})$ 421.6; ${ }^{1} \mathrm{H}$ NMR $\delta(\mathrm{ppm}) 1.25(\mathrm{t}, 3 \mathrm{H}, \mathrm{J}=7.1 \mathrm{~Hz}), 4.17(\mathrm{q}, 2 \mathrm{H}, \mathrm{J}=7.1 \mathrm{~Hz}), 6.46(\mathrm{~d}, 1 \mathrm{H}, \mathrm{J}=15.1 \mathrm{~Hz}, \mathrm{H}-2), 7.20-7.42(\mathrm{~m}, 9 \mathrm{H})$, 7.45-7.55 (m, 2H), $7.80(\mathrm{~d}, 1 \mathrm{H}, \mathrm{J}=15.1 \mathrm{~Hz}, \mathrm{H}-3) ;{ }^{13} \mathrm{C}$ NMR $\delta(\mathrm{ppm}) 14.3,60.6,125.2(\mathrm{C}-2), 127.4,128.2,128.7,129.3(\mathrm{C}-$ 4), 129.5, 129.6, 130.1, 132.4, 136.2, 140.9 (C-3), 144.4 (C-5), 167.1 (C-1).

3.1.8. Preparation of selenoxides 9c-11c. A solution of the diene $(1 \mathrm{mmol})$ in dichloromethane $(5 \mathrm{ml})$ was treated dropwise with $\mathrm{H}_{2} \mathrm{O}_{2}$ (35\% aqueous solution, $\left.0.85 \mathrm{ml}\right)$. The reaction was stirred at room temperature for $1 \mathrm{~h}$. The solution was washed with water, dried, and concentrated under vacuum. The oily product was washed twice with light petroleum and the crude mixture was used directly in the next step.

3.1.8.1. 6-Phenyl-5-phenylseleninylhex-3,5-dien-2-one 9c. Yield 81\%. Major isomer: $60 \%$; ${ }^{1} \mathrm{H}$ NMR $\left(\mathrm{CDCl}_{3}, 200 \mathrm{MHz}\right) \delta$ (ppm) $2.28(\mathrm{~s}, 3 \mathrm{H}), 6.53(\mathrm{~d}, 1 \mathrm{H}, \mathrm{J}=16.5 \mathrm{~Hz}), 7.88(\mathrm{~s}, 1 \mathrm{H}), 7.15-7.80(\mathrm{~m}, 11 \mathrm{H})$. IR (neat): 3057, 2982, 2936, 1715, 1625, $1476,1438,1369,1302,1263,1180,1156,1022,737,690 \mathrm{~cm}^{1}$. Minor isomer: 40\%; ${ }^{1} \mathrm{H}$ NMR $\left(\mathrm{CDCl}_{3}, 200 \mathrm{MHz}\right) \delta(\mathrm{ppm})$ $2.24(\mathrm{~s}, 3 \mathrm{H}), 6.67(\mathrm{~d}, 1 \mathrm{H}, \mathrm{J}=16.2 \mathrm{~Hz}), 7.15-7.65(\mathrm{~m}, 12 \mathrm{H})$.

3.1.8.2. 5-Phenyl-4-phenylseleninylpent-2,4-dieneni-trile 10c. Yield 89\%. Major isomer: $50 \%$; ${ }^{1} \mathrm{H} \mathrm{NMR}\left(\mathrm{CDCl}_{3}, 200 \mathrm{MHz}\right)$ d (ppm) $6.22(\mathrm{~d}, 1 \mathrm{H}, J=16.3 \mathrm{~Hz}), 7.10-7.72(\mathrm{~m}, 12 \mathrm{H})$. Minor isomer: $25 \%$; ${ }^{1} \mathrm{H}$ NMR $\delta(\mathrm{ppm}) 5.45(\mathrm{~d}, 1 \mathrm{H}, J=11.9 \mathrm{~Hz}), 6.81-$ $8.05(\mathrm{~m}, 12 \mathrm{H})$. Minor isomer: $25 \%$; ${ }^{1} \mathrm{H}$ NMR d $\left.(\mathrm{ppm}) 5.92(\mathrm{~d}, 1 \mathrm{H}, \mathrm{J} 1 / 416.6 \mathrm{~Hz}), 6.81-7.72(\mathrm{~m}, 12 \mathrm{H}) \mathrm{J}=7.2 \mathrm{~Hz}\right), 3.03(\mathrm{~d}, 1 \mathrm{H}$, $\mathrm{J}=6.9 \mathrm{~Hz}, \mathrm{OH}), 4.25(\mathrm{~m}, 2 \mathrm{H}), 4.77(\mathrm{ddd}, 1 \mathrm{H}, J=2.6,6.4,6.9 \mathrm{~Hz}, \mathrm{H}-2), 5.72(\mathrm{t}, 1 \mathrm{H}, J=6.4 \mathrm{~Hz}, \mathrm{H}-3), 6.40(\mathrm{dd}, 1 \mathrm{H}, \mathrm{J}=2.6,6.4$ $\mathrm{Hz}, \mathrm{H}-5), 7.17-7.35$ (m, 5H); ${ }^{13} \mathrm{C}$ NMR d (ppm) 14.2, 62.2, 69.0 (C-2), 95.2 (C-3), 98.6 (C-5), 127.1, 127.7, 128.8, 133.1, 173.15 (C-1), 205.43 (C-4). IR (neat): 3457, 2983, 2937, 1954, 1732, 1454, 1371, 1205, 1095, 1025, 862, 770, $699 \mathrm{~cm}^{1}$.

3.1.10. Preparation of tert-butyl- $N$-allenyl carbamates. To a solution of selenide $(1 \mathrm{mmol})$, tert-butyl carbamate $(0.35 \mathrm{~g}, 3$ $\mathrm{mmol})$, triethylamine $(0.8 \mathrm{~g}, 8 \mathrm{mmol})$ in methanol $(3 \mathrm{ml})$ and $N$-bromosuccinimide $(0.35 \mathrm{~g}, 3 \mathrm{mmol})$ were slowly added at $0^{\circ} \mathrm{C}$. The mixture was stirred for $45 \mathrm{~min}$, diluted with ethyl acetate $(10 \mathrm{ml})$, and treated with an aqueous saturated solution of $\mathrm{NaCl}(10 \mathrm{ml})$. After extraction and concentration, the oily residue was chromatographed on silica gel (light petroleum/ethyl acetate: $95 / 5)$.

3.1.10.1. tert-Butyl-N-(4-phenylbut-2,3-dien-1-yl)carbamate 16c. Yield 53\%. ${ }^{1} \mathrm{H}$ NMR $\delta(\mathrm{ppm}) 1.33(9 \mathrm{H}, \mathrm{s}), 3.77(2 \mathrm{H}, \mathrm{m})$, $4.66(1 \mathrm{H}, \mathrm{br} \mathrm{s}), 5.57(1 \mathrm{H}, \mathrm{q}, J=5.6 \mathrm{~Hz}), 6.21(1 \mathrm{H}, \mathrm{dt}, J=3.2,5.6 \mathrm{~Hz}), 7.10-7.26(5 \mathrm{H}, \mathrm{m}) ;{ }^{13} \mathrm{C}$ NMR $\delta(\mathrm{ppm}) 28.5,39.2,79.7$, 93.6, 97.5, 127.0, 127.3, 128.8, 134.0, 155.8, 204.5. Anal. Calcd for $\mathrm{C}_{15} \mathrm{H}_{19} \mathrm{NO}_{2}$ : C, 72.18; H, 7.02; N, 6.11. Found: C, 72.44; $\mathrm{H}, 7.31 ; \mathrm{N}, 5.81$.

3.1.10.2. tert-Butyl- $N$-(5-phenylpent-3,4-dien-2-yl)-carbamate 17c. Yield 48\%. ${ }^{1} \mathrm{H}$ NMR $\delta(\mathrm{ppm}) 1.30(3 \mathrm{H}, \mathrm{d}, \mathrm{J}=6.7 \mathrm{~Hz})$, $1.40(9 \mathrm{H}, \mathrm{s}), 4.32(1 \mathrm{H}, \mathrm{m}), 4.52(1 \mathrm{H}, \mathrm{br} \mathrm{s}), 5.65(1 \mathrm{H}, \mathrm{t}, \mathrm{J}=5.3 \mathrm{~Hz}), 6.24(1 \mathrm{H}, \mathrm{dd}, J=3.1,5.3 \mathrm{~Hz}), 7.13-7.24(5 \mathrm{H}, \mathrm{m}) ;{ }^{13} \mathrm{C}$ NMR $\delta(\mathrm{ppm}) 21.8,(27.03,27.48),{ }^{32} 39.2,52.55,96.9,98.1,125.8,127.2,128.9,133.0,152.0,202.5$. Anal. Calcd for $\mathrm{C}_{16} \mathrm{H}_{21} \mathrm{NO}_{2}$ : C, 74.10; H, 8.16; N, 5.40. Found: C, 74.07; H, 8.32; N, 5.53.

3.1.10.3. tert-Butyl- $N$-(1,4-diphenylbuta-2,3-dien-1-yl)-carbamate 18c. Yield 43\%. ${ }^{1} \mathrm{H}$ NMR $\delta(\mathrm{ppm}) 1.32(9 \mathrm{H}, \mathrm{s}), 5.00(1 \mathrm{H}$, br s), $5.41(1 \mathrm{H}, \mathrm{m}), 5.82(1 \mathrm{H}, \mathrm{t}, J=5.7 \mathrm{~Hz}), 6.40(1 \mathrm{H}, \mathrm{dd}, J=3.5,6.4 \mathrm{~Hz}), 7.10-7.31(10 \mathrm{H}, \mathrm{m}) ;{ }^{13} \mathrm{C} \mathrm{NMR} \mathrm{d}(\mathrm{ppm})(28.03$, 28.46), 39.3, 53.5, 98.2, 98.9, 127.0, 127.1, 127.6, 127.9, 128.4, 128.5, 134.5, 134.6, 155.0, 203.0. Anal. Calcd for $\mathrm{C}_{21} \mathrm{H}_{23} \mathrm{NO}_{2}:$ C, $78.47 ; \mathrm{H}, 7.21 ; \mathrm{N}, 4.36$. Found: $\mathrm{C}, 78.63 ; \mathrm{H}, 7.19 ; \mathrm{N}, 4.32$.

3.1.10.4. Ethyl-2-(tert-butoxycarbonylamino)-5-phenyl-pent-3,4-dienoate 19c. Yield 51\%. ${ }^{1} \mathrm{H}$ NMR $\delta(\mathrm{ppm}) 1.16(3 \mathrm{H}, \mathrm{t}$, $J=7.1 \mathrm{~Hz}), 1.44(9 \mathrm{H}, \mathrm{s}), 4.14-4.25(2 \mathrm{H}, \mathrm{m}), 4.94(1 \mathrm{H}, \mathrm{m}), 5.21(1 \mathrm{H}, \mathrm{br} \mathrm{s}), 5.81(1 \mathrm{H}, \mathrm{m}), 6.40(1 \mathrm{H}, \mathrm{dd}, \mathrm{J}=3.2,6.4 \mathrm{~Hz}), 7.22-$ $7.35(5 \mathrm{H}, \mathrm{m}) ;{ }^{13} \mathrm{C}$ NMR $\delta(\mathrm{ppm})(14.06,14.45),{ }^{32}(28.22,28.31),{ }^{32} 39.2,52.6,(61.81,62.91),{ }^{32} 93.4,(99.20,99.43),{ }^{32} 127.2$, 127.3, 128.8, 133.1, 146.6, 147.7, 155.1, 170.6, 204.3. Anal. Calcd for $\mathrm{C}_{18} \mathrm{H}_{23} \mathrm{NO}_{4}$ : C, 68.11; H, 7.30; N, 4.41. Found: C, $67.88 ; \mathrm{H}, 7.12 ; \mathrm{N}, 4.14$. 
3.1.11. Preparation of $\alpha$-halogenoallenes 20-22. A solution of bromine ( $1.6 \mathrm{ml}, 1 \mathrm{mmol})$ or sulfuryl chloride $(1.35 \mathrm{ml}, 1$ $\mathrm{mmol})$ in hexane was added dropwise to the diene $(1 \mathrm{mmol})$ dissolved in hexane $(10 \mathrm{ml})$ at room temperature. This treatment produces instantaneously a bromo- or chloro-adduct. Their dissolution was carried out in carbon tetrachloride (10 ml) in the presence of a large excess of ethylvinylether $(3 \mathrm{mmol})$. The mixture was stirred for $2 \mathrm{~h}$ under reflux and then concentrated under vacuum. The a-halogenoallene was chromatographed on silica gel (light petroleum/ $\mathrm{CH}_{2} \mathrm{Cl}_{2}$ : 95/5).

3.1.11.1. 4-Chloro-1-phenylbuta-1,2-diene 20c. ${ }^{21}$ Yield 58\%. ${ }^{1} \mathrm{H}$ NMR $\delta$ (ppm) 4.15 (2H, dd, $\left.J=7.5,2.0 \mathrm{~Hz}\right), 5.80(1 \mathrm{H}, \mathrm{m})$, $6.34(1 \mathrm{H}, \mathrm{dt}, J=2.0,6.3 \mathrm{~Hz}), 7.23-7.34(5 \mathrm{H}, \mathrm{m}) ;{ }^{13} \mathrm{C}$ NMR $\delta(\mathrm{ppm}) 42.4,93.5,97.1,127.2,127.7,128.8,133.5,206.5$.

3.1.11.2. 4-Chloro-1-phenylpenta-1,2-diene 21c. Yield 87\%. ${ }^{1} \mathrm{H}$ NMR $\delta(\mathrm{ppm}) 1.68(3 \mathrm{H}, \mathrm{d}, J=6.5 \mathrm{~Hz}), 4.69(1 \mathrm{H}, \mathrm{m}, \mathrm{H}-4)$, $5.87(1 \mathrm{H}, \mathrm{t}, J=6.5 \mathrm{~Hz}, \mathrm{H}-3), 6.39(1 \mathrm{H}, \mathrm{dd}, J=2.1,6.5 \mathrm{~Hz}, \mathrm{H}-1), 7.20-7.35(5 \mathrm{H}, \mathrm{m}) ;{ }^{13} \mathrm{C}$ NMR $\delta(\mathrm{ppm}) 25.1(\mathrm{C}-5), 55.1(\mathrm{C}-4)$, 98.1, 100.0, 127.1, 127.7, 128.9, 133.5, 204.4 (C-2). Anal. Calcd for $\mathrm{C}_{11} \mathrm{H}_{11} \mathrm{Cl}$ : C, 73.94; H, 6.21. Found: C, 74.12; H, 6.13.

3.1.11.3. 4-Bromo-1-phenylpenta-1,2-diene 22c. Yield 61\%. ${ }^{1} \mathrm{H}$ NMR $\delta(\mathrm{ppm}) 1.72(3 \mathrm{H}, \mathrm{d}, \mathrm{J} J=6.7 \mathrm{~Hz}), 5.25(1 \mathrm{H}, \mathrm{m}), 5.52$ $(1 \mathrm{H}, \mathrm{t}, J=6.6 \mathrm{~Hz}), 7.16(1 \mathrm{H}, \mathrm{dd}, J=1.7,6.6 \mathrm{~Hz}), 7.14-7.32(5 \mathrm{H}, \mathrm{m}) ;{ }^{13} \mathrm{C}$ NMR d $(\mathrm{ppm}) 26.4,68.2,100.1,102.6,128.6,129.2$, 131.5, 135.9, 208.2. Anal. Calcd for $\mathrm{C}_{11} \mathrm{H}_{11} \mathrm{Br}$ : C, 59.22; H, 4.97. Found: C, 59.12; H, 5.21.

\section{Acknowledgements}

We thank the region Haute-Normandie (doctoral fellowship to S.R.) for financial support of this research.

\section{References and notes}

1. (a) Wirth, T. Organoselenium Chemistry: Modern develop-ments in Organic Synthesis; Topics in Current Chemistry; Springer: Berlin, Heidelberg, 2000; Vol. 208; (b) Back, T. G. Organoselenium Chemistry: A Practical Approach; Oxford University Press: New York, NY, 1999.

2. Paulmier, C. Selenium Reagents and Intermediates in Organic Synthesis; Pergamon: Oxford, 1986.

3. Liotta, D. C. Organoselenium Chemistry; Wiley: New York, NY, 1987.

4. Patai, S. The Chemistry of Organic Selenium and Tellurium Compounds; Wiley: New York, NY, 1987; Vols. 1 and 2.

5. (a) Wirth, T. Angew. Chem., Int. Ed. Engl. 1995, 34, 1726- 1728; (b) Wirth, T. Tetrahedron 1999, 55, 1-28; (c) Wirth, T. Angew. Chem., Int. Ed. 2000, 39, 3742-3751; (d) Browne, D. M.; Wirth, T. Curr. Org. Chem. 2006, 10, 1893-1903;

(e) Franck, X.; Langlois, E.; Outurquin, F. Synthesis 2007, 719-724.

6. (a) Bowman, W. R.; Bridge, C. F.; Brookes, P. J. Chem. Soc., Perkin Trans. 1 2000, 1-14; (b) Renaud, P.; Andrau, L.; Schenk, L. Synlett 1999, 1462-1464; (c) Zhang, J.; Clive, D. L. J. J. Org. Chem. 1999, 64, 770-779; (d) Ogawa, A.; Ogawa, I.; Obayashi, R.; Umezu, K.; Doi, M.; Hirao, T. J. Org. Chem. 1999, 64, 86-92; (e) Crich, D.; Mo, X. S. J. Am. Chem. Soc. 1998, 120, 8298-8304; (f) Byers, J. H.; Shaughnessy, E. H.; Mackie, T. N. Heterocycles 1998, 48, 2071 and references cited therein.

7. (a) Paulmier, C.; Outurquin, F.; Plaquevent, J. C. Tetrahedron Lett. 1988, 29, 5889-5892; (b) Paulmier, C.; Outurquin, F.; Plaquevent, J. C. Tetrahedron Lett. 1988, 29, 5893-5896;

(c) Outurquin, F.; Paulmier, C. Tetrahedron Lett. 1989, 29, 690-691; (d) Duclos, J. F.; Paulmier, C. Tetrahedron Lett. 1993, 34, 74177420; (e) Ponthieux, S.; Outurquin, F.; Paulmier, C. Tetrahedron Lett. 1995, 36, 6453-6456; (f) Ponthieux, S.; Outurquin, F.; Paulmier, C. Tetrahedron Lett. 1995, 51, 9569-9580.

8. (a) Lerouge, P.; Paulmier, C. Bull. Soc. Chim. Fr. 1985, 1225- 1229; (b) Lerouge, P.; Paulmier, C. Tetrahedron Lett. 1984, 25, 19871990.

9. (a) Bridges, A. J.; Fischer, J. W. Tetrahedron Lett. 1983, 24, 447-450; (b) Liotta, C. L.; Verbicky, J. W., Jr. Tetrahedron Lett. 1985, 26, 1395-1398; (c) Roversi, E.; Monnat, F.; Vogel, P.; Schenk, K.; Roversi, P. Helv. Chim. Acta 2002, 85, 733-760.

10. Bates, G. S.; Fryzuk, M. D.; Stone, C. Can. J. Chem. 1987, 65, 2612-2617.

11. (a) Comasseto, J. V.; Brandt, C. A. Synthesis 1987, 146-149;

(b) Zhu, L.-S.; Huang, Z.-Z.; Huang, X. Tetrahedron 1996, 52, 9819-9822; (c) Ma, Y.; Huang, X. J. Chem. Soc., Perkin Trans. 1 1997, 2953-2955; (d) Cai, M.-Z.; Huang, J.-D.; Peng, C.-Y. J. Organomet. Chem. 2003, 681, 98-101.

12. (a) Lerouge, P.; Paulmier, C. Bull. Soc. Chim. Fr. 1985, 1219- 1224; (b) Paulmier, C.; Lerouge, P. Tetrahedron Lett. 1982, 23, 15571960.

13. (a) Blatcher, P.; Warren, S. J. Chem. Soc., Perkin Trans. 1 1985, 1055-1066; (b) Pearson, W. H.; Lin, K.-C.; Poon, Y.-F. J. Org. Chem. 1989, 54, 5814-5819; (c) Voyle, M.; Kyler, K. S.; Arseniyadis, S.; Dunlap, N. K.; Watt, D. S. J. Org. Chem. 1983, 48, 470476.

14. Squillacote, M. E.; Liang, F. J. Org. Chem. 2005, 70, 6564- 6573. 
15. (a) The Chemistry of Ketenes, Allenes, and Related Compounds; Patai, S., Ed.; John Wiley and Sons: Chichester, UK, 1980; (b) Brandsma, L.; Verkruijsse, H. D. Synthesis of Acetylenes, Allenes, and Cumulenes; Elvesier: Amsterdam, 1981; (c) The Chemistry of the Allenes; Landor, S. R., Ed.; Academic: London, 1983; (d) Schuster, H.; Coppola, G. Allenes in Organic Synthesis; John Wiley and Sons: New York, NY, 1984; (e) Pasto, D. J. Tetrahedron 1984, 40, 2805- 2827.

16. Modern Allene Chemistry; Krause, N., Hashmi, A. S. K., Eds.; Wiley-VCH: Weinheim, 2004.

17. (a) Yamamoto, Y.; Radhakrishnan, U. Chem. Soc. Rev. 1999, 28, 199-207; (b) Hashmi, A. S. K. Angew. Chem., Int. Ed. 2000, 39, 35903593; (c) Zimmer, R.; Dinesh, C. U.; Nandanan, E.; Khan, F. A. Chem. Rev. 2000, 100, 3067-3125.

18. Ohno, H. Chem. Pharm. Bull. 2005, 53, 1211-1226.

19. (a) Krause, N.; Laux, M.; Hoffmann-R€oder, A. Tetrahedron Lett. 2000, 41, 9613-9616; (b) Krause, N.; Hoffmann-R€oder, A. Tetrahedron 2004, 60, 11671-11694.

20. Perepelkin, O. V.; Cherkasov, L. N.; Korner, V. A.; Bal'yan, K. V.; Petrov, A. A. Zh. Obshch. Khim. 1965, 35, 574-578.

21. Cowie, J. S.; Landor, P. D.; Landor, S. R. J. Chem. Soc., Perkin Trans. 1 1973, 720-724.

22. Alexakis, A.; Marek, I.; Mangeney, P.; Normant, J. F. Tetrahedron 1991, 47, 1677-1696.

23. (a) Marshall, J. A.; Robinson, E. D.; Zapata, A. J. Org. Chem. 1989, 54, 5854-5855; (b) Marshall, J. A.; Wang, X. J. J. Org. Chem. 1990, 55, 2995-2996; (c) Marshall, J. A.; Wang, X. J.

J. Org. Chem. 1991, 56, 4913-4918.

24. Katsuhira, T.; Harada, T.; Oku, A. J. Org. Chem. 1994, 59, 4010-4014.

25. (a) Vinson, N. A.; Day, C. S.; Welker, M. E. Organometallics 2000, 19, 4356-4368; (b) Shi, L.; Xia, W.; Yang, J.; Wen, X.; Huang, Y. Z. Tetrahedron Lett. 1987, 28, 2155-2158.

26. Horvath, A.; B€ackvall, J.-E. J. Org. Chem. 2001, 66, 8120- 8126.

27. Trost, B. M.; Jonasson, C.; Wuchrer, M. J. Am. Chem. Soc. 2001, 123, 12736-12737.

28. (a) Halliday, R. P.; Davis, C. S.; Heotis, J. P.; Pals, D. T.; Watson, E. J.; Bickerton, R. K. J. Pharm. Sci. 1968, 57, 430- 433; (b) Krantz, A.; Kokel, B.; Sachdeva, Y. P.; Salach, J.; Claesson, A.; Sahlberg, C. Drug Action and Design: Mechanism-based Enzyme Inhibitors; Kalman, Ed.; Elsevier: North Holland, 1979; p 145.

29. (a) Monoamine Oxidase and its Inhibitors; Ciba Foundation Symposium 39; Elsevier: North Holland, 1976; (b) Singer,

T. P.; Von Korf, R. W.; Murphy, D. L. Monoamine Oxidase, Structure Function and Altered Functions; Academic: New York, NY, 1979.

30. (a) Frankhauser, J. E.; Peevey, R. M.; Hopkins, P. B. Tetrahedron Lett. 1984, 25, 15-18; (b) Shea, R. G.; Fitzner, J. N.; Frankhauser, J. E.; Hopkins, P. B. J. Org. Chem. 1984,

49, 3647-3650; (c) Fitzner, J. N.; Shea, R. G.; Frankhauser, E.; Hopkins, P. B. J. Org. Chem. 1985, 50, 417-419; (d) Spaltenstein, A.; Carpino, P. A.; Hopkins, P. B. Tetrahedron Lett. 1986, 27, 147-150; (e) Shea, R. G.; Fitzner, J. N.; Frankhauser, J. E.; Spaltenstein, P. A.; Peevey, R. M.; Pratt,

D. V.; Tenge, B. J.; Hopkins, P. B. J. Org. Chem. 1986, 51, 5243-5252; (f) Spaltenstein, A.; Carpino, P. A.; Miyake, F.; Hopkins, P. B. J. Org. Chem. 1987, 52, 3759-3766.

31. Krijnen, E. S.; Zuilhof, H.; Lodder, G. J. Org. Chem. 1994, 59, 8139-8150.

32. Peaks appeared as doublet due to diastereomeric mixtures. 